\title{
MIXED FINITE ELEMENT APPROXIMATION OF AN MHD PROBLEM INVOLVING CONDUCTING AND INSULATING REGIONS: THE 2D CASE
}

\author{
Jean Luc Guermond ${ }^{1}$ And Peter D. Minev ${ }^{2}$
}

\begin{abstract}
We show that the Maxwell equations in the low frequency limit, in a domain composed of insulating and conducting regions, has a saddle point structure, where the electric field in the insulating region is the Lagrange multiplier that enforces the curl-free constraint on the magnetic field. We propose a mixed finite element technique for solving this problem, and we show that, under mild regularity assumption on the data, Lagrange finite elements can be used as an alternative to edge elements.
\end{abstract}

Mathematics Subject Classification. 65N35, 65N25, 65F05, 35J05.

Received: October 25, 2001, Revised: March 3, 2002.

\section{INTRODUCTION}

The problem under consideration in the present paper stems from the so-called Dynamo problem which has attracted the interest of astrophysicists for a long time. Though we shall hereafter restrict ourselves to the electromagnetic part of the problem, let us briefly restate the whole setting. In astrophysical situations like stars or planets with a liquid core, a magnetic field is sustained by nonlinear interactions with large-scale movements of a conducting fluid. The fluid may be plasma in the stellar case, molten iron in the planetary case, or liquid gallium or sodium in experimental setups that try to reproduce the dynamo effect. One important aspect of the problem is that the magnetic field develops in a non-homogeneous medium: there are conducting regions and insulation regions. The insulating region may be vacuum, rock, air, etc. We refer to Moffatt [16] for a survey on the physical aspects of problem.

The equations that model the dynamo effect are the incompressible Navier-Stokes equations and the Maxwell equations with the displacement-currents neglected, the two set of equations being coupled via the Lorentz force and the Ohm's law. Denoting by $\mathbf{u}, p, \mathbf{E}$, and $\mathbf{H}$ the velocity, pressure, electric and magnetic fields, and denoting

\footnotetext{
Keywords and phrases. Finite element method, Magnetohydrodynamics.

1 LIMSI (CNRS-UPR 3152), BP 133, 91403, Orsay, France. e-mail: guermond@limsi.fr

2 Department of Mathematical Sciences, University of Alberta Edmonton, Alberta, Canada T6G 2G1, Canada.

e-mail: minev@ualberta.ca
} 
by $\mathbf{f}$ and $\mathbf{j}$ given source terms, the sets of equations are as follows:

$$
\begin{aligned}
& \left\{\begin{array}{l}
\partial_{t} \mathbf{u}+(\mathbf{u} \cdot \nabla) \mathbf{u}-\eta \nabla^{2} \mathbf{u}+\nabla p=(\nabla \times \mathbf{H}) \times \mu \mathbf{H}+\mathbf{f} \text { in } \Omega_{c} \\
\nabla \cdot \mathbf{u}=0 \\
\left.\mathbf{u}\right|_{\partial \Omega_{c}=\mathbf{a}}
\end{array}\right. \\
& \left\{\begin{array}{lr}
\partial_{t}(\mu \mathbf{H})=-\nabla \times \mathbf{E} & \text { in } \Omega_{c} \\
\nabla \times \mathbf{H}=\sigma(\mathbf{E}+\mathbf{u} \times(\mu \mathbf{H}))+\mathbf{j} & \text { in } \Omega_{c} \\
\nabla \times \mathbf{H}=0 & \text { in } \Omega_{v} \\
\nabla \cdot(\epsilon \mathbf{E})=0 & \text { in } \Omega_{v}, \\
\mathbf{H} \times\left.\mathbf{n}\right|_{\Gamma}=0 & \\
\left.\mathbf{E} \cdot \mathbf{n}\right|_{\Gamma_{v}}=0, &
\end{array}\right.
\end{aligned}
$$

where $\Omega_{c}$ denotes the domain occupied by the conducting fluid, $\Omega_{v}$ is the domain of the non-conducting medium, and $\Omega=\Omega_{c} \bigcup \Omega_{v}$ is the total domain, see Figure 1. The subscripts $c$ and $v$ stand for conductor and vacuum respectively. The magnetic permeability $\mu=\mu(\mathbf{x})$, the electric permittivity $\epsilon=\epsilon(\mathbf{x})$, and conductivity $\sigma=\sigma(\mathbf{x})$ are supposed to be positive functions of $\mathbf{x}$ only.

The main difficulty with this form of the MHD equations comes from the presence of the non-conducting medium where $\sigma$ is zero. In this region the magnetic field $\mathbf{H}$ must be curl-free and the electric induction divergence-free. If it was not for this condition (in other words, if $\Omega=\Omega_{c}$ ), then the electric field could be eliminated from the system of equations as done in most of the studies dealing with the MHD equations and the resulting equations would be parabolic, thus posing no particular difficulty to be approximated numerically (see e.g. $[1,9,21])$. Most of the existing numerical works dedicated to the dynamo either assume that the conducting region has perfectly conducting walls ("ideal" boundary) or enforce on the interface $\Sigma$ an ad hoc boundary condition on $\mathbf{H}$ so that the problem can be restricted to the conducting region only. Few studies consider the MHD equations with "non-ideal" boundaries and most of them are either restricted to steady situations (see, for example [17] and the references therein), or consider simple geometries for the conducting region like infinite cylinders or spheres so that the exterior problem can be solved analytically $[7,13,14]$. In the stationary case with "non-ideal" boundary, the magnetic field is usually eliminated by means of the Biot-Savart law as suggested in $[17]$.

In the present paper we restrict ourselves to the electromagnetic part of the problem, namely (1.2), and we discuss the possibility of approximating the problem with neither the magnetic nor the electric field eliminated from the system. Our goal is to show that (1.2) is a saddle point problem where the electric field in the insulating region is the Lagrange multiplier for enforcing the magnetic field to be curl-free. We propose a mixed finite element technique for solving this problem in its saddle point form, and we show that, under mild regularity assumption on the data, Lagrange finite elements can be used as an alternative to edge elements (sometimes also called Whithney or Nédélec finite elements, see $[3,19])$.

Actually there are two difficulties associated with the presence of insulating regions: the first one, already mentioned above, is that it gives to the problem a saddle point structure, the second one is that the electric induction, $\epsilon \mathbf{E}$, must be solenoidal in the non-conduction region. That is to say, the Lagrange multiplier, E, is subject to the additional constraint $\left.\nabla \cdot(\epsilon \mathbf{E})\right|_{\Omega_{v}}=0$. To avoid mixing at once these two issues and to emphasize the saddle point character of the problem, we restrict ourselves, in the present paper, to the $2 \mathrm{D}$ situation where the electric field is perpendicular to the plane and can be considered to be a scalar. As a result, the solenoidality constraint on $\epsilon \mathbf{E}$ is automatically satisfied. The second issue, namely the treatment of the constraint $\left.\nabla \cdot(\epsilon \mathbf{E})\right|_{\Omega_{v}}=0$ in the full 3D setting will be reported in a separate paper [11].

The paper is organized as follows. In the next section we discuss the saddle point structure of the problem and its wellposedness. We introduce the basic formulation and two stabilized formulations. In Section 3 , we discuss the finite element discretization of the problem in its saddle point form. We review possible choices of admissible elements, and we derive a priori error estimates. In Section 4 we present some numerical results using $\mathbb{P}_{2} / \mathbb{P}_{1}$ triangular elements. 


\section{The SADDLE POINT STRUCTURE}

\subsection{Preliminaries}

Let us define precisely the domain geometry. $\Omega$ is a Lipschitz, open, bounded, connected domain in $\mathbb{R}^{2}$. We assume that $\Omega$ is non-trivially partitioned into two Lipschitz subdomains $\Omega_{v}$ and $\Omega_{c}$ so that

$$
\bar{\Omega}=\overline{\Omega_{c}} \cup \overline{\Omega_{v}}, \quad \Omega_{c} \cap \Omega_{v}=\emptyset .
$$

Consequently, we introduce

$$
\Gamma_{c}=\partial \Omega \cap \partial \Omega_{c}, \quad \Gamma_{v}=\partial \Omega \cap \partial \Omega_{v}, \quad \Sigma=\partial \Omega_{c} \cap \partial \Omega_{v}, \quad \Gamma=\partial \Omega=\Gamma_{v} \cup \Gamma_{c} .
$$

In astrophysical applications we usually have $\Gamma_{c}=\emptyset$, but we shall not make this hypothesis. Three possible partitioning of $\Omega$ are shown in Figure 1 .
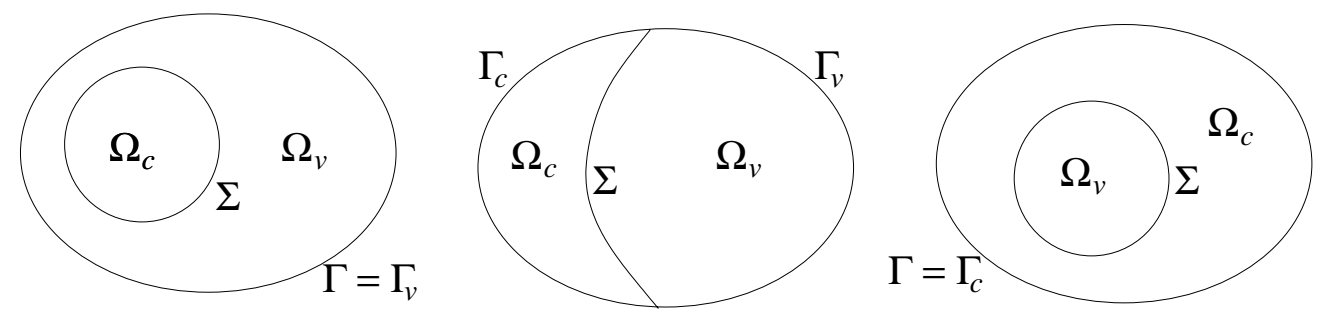

Figure 1. Three possible settings for the domain.

We denote by $\mathbb{L}^{2}(\Omega)$ (resp. $\mathbb{H}^{1}(\Omega)$ ) the space of vector valued functions whose components are in $L^{2}(\Omega)$ (resp. in $H^{1}(\Omega)$ ). The norms of the Sobolev spaces $W^{m, p}(\Omega)$ and $\mathbb{W}^{m, p}(\Omega)$ are denoted by $\|\cdot\|_{m, p, \Omega}$ with no distinction between scalar and vector-valued functions. The norms of $H^{s}(\Omega)$ and $\mathbb{H}^{s}(\Omega)$ are denoted by $\|\cdot\|_{s, \Omega}$. For any given subdomain $\Omega_{s} \subset \Omega$, we denote by $(., .)_{\Omega_{s}}$ the $L^{2}$-scalar product on $\Omega_{s}$. Throughout this paper we denote by $c$ a generic constant that does not depend on the mesh size, $h$, but whose value may change at each occurrence.

Since the Navier-Stokes equations (1.1) are well studied, we shall concentrate in this paper on the Maxwell part of the problem, i.e. (1.2). Therefore, we suppose that $\mathbf{u}$ is a known, smooth enough vector field. To emphasize the saddle point structure of the problem without having to bother about the additional constraint $\left.\nabla \cdot(\epsilon \mathbf{E})\right|_{\Omega_{v}}=0$, we restrict ourselves to the $2 \mathrm{D}$ situation where the electric field is a scalar. Therefore, we denote the electric field by $E$ and the corresponding test functions by $e$. As a result, the constraints $\left.\nabla \cdot(\epsilon \mathbf{E})\right|_{\Omega_{v}}=0$ and $\mathbf{E} \cdot \mathbf{n}=0$ are automatically satisfied. Of course the condition $\left.\nabla \cdot(\epsilon \mathbf{E})\right|_{\Omega_{v}}=0$ is a very important element in the structure of the problem, and we show how it can be taken into account within the framework developed herein in a separate paper [11]. The set of equations we study in the present paper is as follows

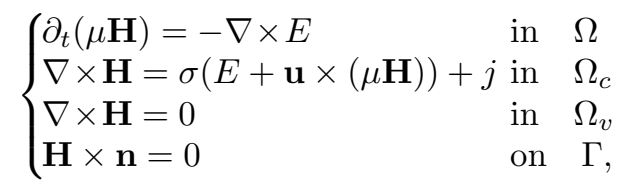

where we adopt the following conventions:

$$
\nabla \times \mathbf{H}=\partial_{1} H_{2}-\partial_{2} H_{1}, \quad \nabla \times E=\left(\partial_{2} E,-\partial_{1} E\right), \quad \mathbf{u} \times(\mu \mathbf{H})=\mu\left(u_{1} H_{2}-u_{2} H_{1}\right)
$$




\subsection{The basic formulation}

To obtain a weak form of the system (2.1) we multiply the first equation by test functions and we integrate over $\Omega$ :

$$
\left(\mu \partial_{t} \mathbf{H}, \mathbf{b}\right)_{\Omega}+(\nabla \times E, \mathbf{b})_{\Omega}=0, \quad \forall \mathbf{b}
$$

where $\mathbf{b}$ are the test functions to be chosen later and $(., .)_{\Omega}$ denotes the $L^{2}$-scalar product on $\Omega$. Now we integrate by parts the second term in the equation, and assuming that the test functions $\mathbf{b}$ satisfy the same essential boundary condition as $\mathbf{H}$, we obtain

$$
\left(\mu \partial_{t} \mathbf{H}, \mathbf{b}\right)_{\Omega}+(E, \nabla \times \mathbf{b})_{\Omega}=0, \quad \forall \mathbf{b}
$$

The second integral in this equation can be represented as a sum of the integrals over $\Omega_{c}$ and $\Omega_{v}$. Then taking into account that in the conducting medium $\Omega_{c}$ we have

$$
E=\frac{1}{\sigma}(\nabla \times \mathbf{H}-j)-\mathbf{u} \times(\mu \mathbf{H})
$$

we obtain

$$
\left(\mu \partial_{t} \mathbf{H}, \mathbf{b}\right)_{\Omega}+\left(\frac{1}{\sigma} \nabla \times \mathbf{H}, \nabla \times \mathbf{b}\right)_{\Omega_{c}}-(\mathbf{u} \times \mu \mathbf{H}, \nabla \times \mathbf{b})_{\Omega_{c}}+(E, \nabla \times \mathbf{b})_{\Omega_{v}}=\left(\frac{1}{\sigma} j, \nabla \times \mathbf{b}\right)_{\Omega_{c}}, \quad \forall \mathbf{b} .
$$

Now, we enforce the constraint $\nabla \times \mathbf{H}=0$ in $\Omega_{v}$ as follows

$$
(\nabla \times \mathbf{H}, e)_{\Omega_{v}}=0 \quad \forall e
$$

To formalize our problem, we introduce an appropriate functional setting as follows:

$$
\left\{\begin{array}{l}
\mathbf{X}_{1}=\left\{\mathbf{b} \in \mathbb{L}^{2}(\Omega), \nabla \times \mathbf{b} \in \mathbb{L}^{2}(\Omega), \mathbf{b} \times \mathbf{n}_{\mid \Gamma}=0\right\} \\
\mathbf{V}=\left\{\mathbf{b} \in \mathbf{X}_{1}, \nabla \times \mathbf{b}=0 \text { in } \Omega_{v}\right\} \\
\mathbf{L}=\left\{\mathbf{b} \in \mathbb{L}^{2}(\Omega), \nabla \times \mathbf{b}=0 \text { in } \Omega_{v}, \mathbf{b} \times\left.\mathbf{n}\right|_{\Gamma_{v}}=0\right\} \\
M=L^{2}\left(\Omega_{v}\right) \\
W(0, T ; \mathbf{V})=\left\{\mathbf{b}(t) \in L^{2}(0, T ; \mathbf{V}), \mathbf{b}_{t}(t) \in L^{2}\left(0, T ; \mathbf{V}^{\prime}\right)\right\}
\end{array} .\right.
$$

We introduce also the bilinear form $a_{1} \in \mathcal{L}\left(\mathbf{X}_{1} \times \mathbf{X}_{1} ; \mathbb{R}\right)$, such that

$$
a_{1}\left(\mathbf{b}, \mathbf{b}^{\prime}\right)=\left(\frac{1}{\sigma} \nabla \times \mathbf{b}, \nabla \times \mathbf{b}^{\prime}\right)_{\Omega_{c}}-\left(\mathbf{u} \times \mu \mathbf{b}, \nabla \times \mathbf{b}^{\prime}\right)_{\Omega_{c}} .
$$

To ensure wellposedness, we make the following assumptions on the electric conductivity and the magnetic permeability: $\sigma \in L^{\infty}\left(\Omega_{c}\right), \mu \in L^{\infty}(\Omega)$ and $\inf _{x \in \Omega_{c}} \frac{1}{\sigma} \geq \frac{1}{\sigma_{0}}>0$, inf $x \in \Omega \geq \mu_{0}>0$. Then the bilinear form $a_{1}$ satisfies a Gårding-like inequality.

Lemma 2.1. Under the assumptions above on $\sigma$ and $\mu$, there are $\gamma \geq 0$ and $c>0$ s.t.

$$
\forall \mathbf{H} \in \mathbf{V}, \quad a_{1}(\mathbf{H}, \mathbf{H})+\gamma\|\mathbf{H}\|_{0, \Omega}^{2} \geq c\|\mathbf{H}\|_{\mathbf{X}_{1}}^{2} .
$$


Proof. Let us set $c(\mathbf{u}, \mu)=\|\mathbf{u}\|_{0, \infty, \Omega}\|\mu\|_{0, \infty, \Omega}$ and $\gamma=c(\mathbf{u}, \mu)^{2} \sigma_{0}$. Then for all $\mathbf{H} \in \mathbf{V}$ we have the following:

$$
\begin{aligned}
a_{1}(\mathbf{H}, \mathbf{H})+\gamma\|\mathbf{H}\|_{0, \Omega}^{2} & \geq \frac{1}{\sigma_{0}}\|\nabla \times \mathbf{H}\|_{0, \Omega_{c}}^{2}-c(\mathbf{u}, \mu)\|\mathbf{H}\|_{0, \Omega_{c}}\|\nabla \times \mathbf{H}\|_{0, \Omega_{c}}+\gamma\|\mathbf{H}\|_{0, \Omega}^{2} \\
& \geq \frac{1}{\sigma_{0}}\|\nabla \times \mathbf{H}\|_{0, \Omega_{c}}^{2}-\frac{1}{2 \sigma_{0}}\|\nabla \times \mathbf{H}\|_{0, \Omega_{c}}^{2}+\left(\gamma-c(\mathbf{u}, \mu)^{2} \frac{\sigma_{0}}{2}\right)\|\mathbf{H}\|_{0, \Omega}^{2} \\
& \geq \frac{1}{2 \sigma_{0}}\left(\|\nabla \times \mathbf{H}\|_{0, \Omega_{c}}^{2}+\|\nabla \times \mathbf{H}\|_{0, \Omega_{v}}^{2}\right)+c(\mathbf{u}, \mu)^{2} \frac{\sigma_{0}}{2}\|\mathbf{H}\|_{0, \Omega}^{2} \\
& \geq \min \left(\frac{1}{2 \sigma_{0}}, c(\mathbf{u}, \mu)^{2} \frac{\sigma_{0}}{2}\right)\|\mathbf{H}\|_{\mathbf{X}_{1}}^{2} .
\end{aligned}
$$

With the definitions above in hand, our problem now takes the following weak form: For $j \in L^{2}\left(0, T ; L^{2}(\Omega)\right)$ and $\mathbf{H}_{0} \in \mathbf{L}$,

$$
\begin{cases}\text { Find } \mathbf{H} \in W\left(0, T ; \mathbf{X}_{1}\right) \text { and } E \in \mathcal{D}^{\prime}(0, T ; M) \text { s.t. }, \forall \mathbf{b} \in \mathbf{X}_{1}, & \forall e \in M \\ \left(\mu \partial_{t} \mathbf{H}, \mathbf{b}\right)_{\Omega}+a_{1}(\mathbf{H}, \mathbf{b})+(E, \nabla \times \mathbf{b})_{\Omega_{v}}=\left(\frac{1}{\sigma} j, \nabla \times \mathbf{b}\right)_{\Omega_{c}} & \text { in } \mathcal{D}^{\prime}(0, T) \\ (\nabla \times \mathbf{H}, e)_{\Omega_{v}}=0 & \text { in } L^{2}(0, T) .\end{cases}
$$

Note that this formulation is remarkably similar to the weak formulation of the Stokes equations. In fact, the electric field $E$ comes into play into the non-conducting medium only, and it appears to be the Lagrange multiplier for the imposition of the linear constraint $\nabla \times \mathbf{H}=0$ in $\Omega_{v}$.

Theorem 2.1. Problem (2.2) is well-posed.

Proof. Let us consider the following problem

$$
\left\{\begin{array}{l}
\text { Find } \mathbf{H} \in W(0, T ; \mathbf{V}) \text { such that } \\
\left(\mu \partial_{t} \mathbf{H}, \mathbf{b}\right)_{\Omega}+a_{1}(\mathbf{H}, \mathbf{b})=\left(\frac{1}{\sigma} j, \nabla \times \mathbf{b}\right)_{\Omega_{c}}, \quad \forall \mathbf{b} \in \mathbf{V}, \text { in } \mathcal{D}^{\prime}(0, T) \\
\left.\mathbf{H}\right|_{t=0}=\mathbf{H}_{0} .
\end{array}\right.
$$

Owing to the coercivity property stated in Lemma 2.1, Lions' theorem (see e.g. [4, p .218], [15, pp. 253-258]) ensures that problem (2.3) has a unique solution $\mathbf{H} \in W(0, T ; \mathbf{V})$ which satisfies the initial condition due to the fact that $W(0, T ; \mathbf{V}) \subset \mathcal{C}^{0}(0, T ; \mathbf{L})$.

For $E$ we consider the following problem

$$
\left\{\begin{array}{l}
\text { Find } E \in \mathcal{D}^{\prime}(0, T ; M) \text { such that } \forall \mathbf{b} \in \mathbf{X}_{1} \\
(E, \nabla \times \mathbf{b})_{\Omega_{v}}=-\left(\mu \partial_{t} \mathbf{H}, \mathbf{b}\right)_{\Omega}-a_{1}(\mathbf{H}, \mathbf{b})+\left(\frac{1}{\sigma} j, \nabla \times \mathbf{b}\right)_{\Omega} .
\end{array}\right.
$$

Let us introduce the linear form $\phi \in \mathbf{X}_{1}^{\prime}$ so that for all $\mathbf{b}$ in $\mathbf{X}_{1}$ we have

$$
\langle\phi(\mathbf{H}), \mathbf{b}\rangle=-\left(\mu \partial_{t} \mathbf{H}, \mathbf{b}\right)_{\Omega}-a_{1}(\mathbf{H}, \mathbf{b})+\left(\frac{1}{\sigma} j, \nabla \times \mathbf{b}\right)_{\Omega} .
$$

It is clear that due to the definition of $\mathbf{H}$, the restriction of $\phi(\mathbf{H})$ to $\mathbf{V}$ is zero. That is, $\phi(\mathbf{H})$ is in the polar set of $\mathbf{V}$, i.e. $\phi(\mathbf{H}) \in \mathbf{V}^{\perp}$. Let us define the linear operator $R: \mathbf{X}_{1} \ni \mathbf{b} \longmapsto \nabla \times\left.\mathbf{b}\right|_{\Omega_{v}} \in M$. It is clear that $\mathbf{V}$ is the null space of $R$, i.e. $\mathbf{V}=\mathcal{N}(R)$. As a result we have $\phi(\mathbf{H}) \in \mathbf{V}^{\perp}=\mathcal{N}(R)^{\perp}$, that is $\phi(\mathbf{H})$ is in $\overline{\mathcal{R}\left(R^{t}\right)}$, i.e. the closure of the range of the transpose of $R$. Owing to Lemma 2.2 and Banach's closed range theorem, we infer 
that there is some $E$ in $\mathcal{D}^{\prime}(0, T ; M)$ so that $R^{t}(E)=-\phi(\mathbf{H})$, the equality holding in $\mathcal{D}^{\prime}(0, T)$. In other words, there is $E \in \mathcal{D}^{\prime}(0, T ; M)$ so that for all $\mathbf{b}$ in $\mathbf{X}_{1}$,

$$
\langle\phi(\mathbf{H}), \mathbf{b}\rangle=-\left\langle R^{t}(E), \mathbf{b}\right\rangle=-(E, R(\mathbf{b}))_{\Omega_{v}}=-(E, \nabla \times \mathbf{b})_{\Omega_{v}}, \quad \text { in } \mathcal{D}^{\prime}(0, T) .
$$

This completes the proof.

Lemma 2.2. The operator $R: \mathbf{X}_{1} \ni \mathbf{b} \longmapsto \nabla \times \mathbf{b} \in M$, is surjective.

Proof. Let $e \in M$ and consider the following boundary value problem

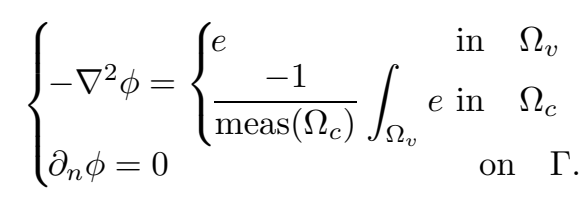

This problem is wellposed in $H^{1}(\Omega) / \mathbb{R}$ and $\|\phi\|_{1, \Omega} \leq c\left\|e_{h}\right\|_{M}$. Then setting $\mathbf{b}=\nabla \times \phi$ we obtain $R \mathbf{b}=e$ in $\Omega_{v}$, and

$$
\begin{aligned}
\|\mathbf{b}\|_{0, \Omega} & \leq\|\phi\|_{1, \Omega} \leq c\|e\|_{M} \\
\|\nabla \times \mathbf{b}\|_{0, \Omega} & =\left\|\nabla^{2} \phi\right\|_{0, \Omega} \leq c\|e\|_{M} .
\end{aligned}
$$

Since also $\mathbf{b} \times\left.\mathbf{n}\right|_{\Gamma}=0$ then $\mathbf{b} \in \mathbf{X}_{1}$ and $\|\mathbf{b}\| \mathbf{x}_{1} \leq \sqrt{c^{2}+1}\|e\|_{M}$. This completes the proof.

Remark 2.1. Note that this lemma implies that the range of $R$ and the range of $R^{t}$ are closed. Note also that from this lemma we infer that there exists $\beta>0$ such that

$$
\sup _{\mathbf{b} \in \mathbf{X}_{1}} \frac{(\nabla \times \mathbf{b}, e)}{\|\mathbf{b}\|_{\mathbf{X}_{1}}} \geq \beta\|e\|_{M}, \quad \forall e \in M
$$

Remark 2.2. When it comes to approximate the problem above, two strategies are possible: one can approximate either the problem (2.2) or the problem (2.3). The approaches based on edge finite elements consist in working with formulation (2.3) where the field $E$ is completely eliminated and the constraint $\nabla \times \mathbf{H}=0$ is enforced in an essential way by seeking $\mathbf{H}$ in $\mathbf{V}$. It is possible to build internal approximations in $\mathbf{V}$ by using gradients of continuous Lagrange finite elements with support in $\Omega_{v}$ see e.g. Bossavit [3]. Contrary to these techniques, the originality of the approach herein consists of retaining the field $E$ in $\Omega_{v}$ as a Lagrange multiplier for enforcing the curl-free constraint on $\mathbf{H}$ in $\Omega_{v}$ only weakly. To the best of our knowledge, the literature does not discuss numerical methods based directly on formulation (2.2).

\subsection{A partially stabilized formulation}

As it will be clear in Section 3.1, when it come to approximate (2.2) with Lagrange finite elements, the fact that $a_{1}$ satisfies a Gårding inequality only in $\mathbf{V}$ is not sufficient to prove reasonable $a$ priori error estimates. To enhance the stability of the formulation we introduce the following new bilinear form

$$
a_{1 s}\left(\mathbf{b}, \mathbf{b}^{\prime}\right)=\left(\frac{1}{\tilde{\sigma}} \nabla \times \mathbf{b}, \nabla \times \mathbf{b}^{\prime}\right)_{\Omega}-\left(\mathbf{u} \times(\mu \mathbf{b}), \nabla \times \mathbf{b}^{\prime}\right)_{\Omega_{c}},
$$

where we have defined $\tilde{\sigma}$ to be a smooth extension of $\sigma$ on the whole domain $\Omega$ so that for all $x \in \Omega$, $\inf _{y \in \Omega_{c}} \sigma(y) \leq \tilde{\sigma}(x) \leq \sup _{y \in \Omega_{c}} \sigma(y)$. It is clear that $a_{1 s} \in \mathcal{L}\left(\mathbf{X}_{1} \times \mathbf{X}_{1} ; \mathbb{R}\right)$, and by repeating the arguments in the proof of Lemma 2.1, we can prove 
Lemma 2.3. Under the assumptions of Lemma 2.1, and if $\tilde{\sigma}$ satisfies the assumption above, then there are $\gamma \geq 0$ and $c>0$ s.t.

$$
\forall \mathbf{H} \in \mathbf{X}_{1}, \quad a_{1 s}(\mathbf{H}, \mathbf{H})+\gamma\|\mathbf{H}\|_{0, \Omega}^{2} \geq c\|\mathbf{H}\|_{\mathbf{X}_{1}}^{2}
$$

Note that contrary to Lemma 2.1 , the coercivity now holds for all functions in $\mathbf{X}_{1}$.

We now consider the following problem: For $j \in L^{2}\left(0, T ; L^{2}(\Omega)\right)$ and $\mathbf{H}_{0} \in \mathbf{L}$,

$$
\left\{\begin{array}{l}
\text { Find } \mathbf{H} \in W\left(0, T ; \mathbf{X}_{1}\right) \text { and } E \in \mathcal{D}^{\prime}(0, T ; M) \text { s.t. }, \forall \mathbf{b} \in \mathbf{X}_{1}, \quad \forall e \in M \\
\left(\mu \partial_{t} \mathbf{H}, \mathbf{b}\right)_{\Omega}+a_{1 s}(\mathbf{H}, \mathbf{b})+(E, \nabla \times \mathbf{b})_{\Omega_{v}}=\left(\frac{1}{\sigma} j, \nabla \times \mathbf{b}\right)_{\Omega_{c}} \\
(\nabla \times \mathbf{H}, e)_{\Omega_{v}}=0 \quad \text { in } L^{2}(0, T) .
\end{array}\right.
$$

Theorem 2.2. Problem (2.6) is well-posed and the solutions to (2.2) and (2.6) are identical.

Proof. Repeat the arguments of the proof of Theorem 2.1.

\subsection{A fully stabilized formulation}

As they stand now, neither formulation (2.2) nor (2.6) permit to establish easily long-time exponential stability, since the bilinear forms $a_{1}$ and $a_{1 s}$ are not strongly coercive in $\mathbf{X}_{1}$. Furthermore, they yield stability on the magnetic field $\mathbf{H}$ only in the $\mathbf{X}_{1}$-norm. As a result, any reasonable approximation scheme based on this formulation should yield convergence on this quantity only in the $\mathbf{X}_{1}$-norm. That is, we should not expect convergence on $\mathbf{H}$ in $\mathbb{H}^{1}(\Omega)$ from this formulation. For instance, no long-time stability on the divergence of $\mu \mathbf{H}$ is guaranteed a priori. We propose now an alternative formulation that solves some of these problems if the magnetic permeability is constant or a very smooth function.

Let us assume from now on that $\mu$ is smooth. We assume also that the initial data $\mu \mathbf{H}_{0}$ is divergence-free, so that, owing to the equation $\partial_{t}(\mu \mathbf{H})=-\nabla \times E$, we infer that $\nabla \cdot(\mu \mathbf{H})=0$ for all times. As a result, we should expect some bound in the $\mathbb{H}^{1}(\Omega)$-norm on $\mathbf{H}$, at least locally, since we have an a priori bound on $\nabla \times \mathbf{H}$ in $\Omega_{c}$ and we know that $\nabla \times \mathbf{H}=0$ in $\Omega_{v}, \nabla \cdot(\mu \mathbf{H})=0$ in $\Omega$, and $\mathbf{H} \times \mathbf{n}=0$ on $\Gamma$.

To formalize these ideas, we introduce the new space

$$
\mathbf{X}_{2}=\left\{\mathbf{b} \in \mathbb{L}^{2}(\Omega), \nabla \cdot(\mu \mathbf{b}) \in \mathbb{L}^{2}(\Omega), \nabla \times \mathbf{b} \in \mathbb{L}^{2}(\Omega), \mathbf{b} \times\left.\mathbf{n}\right|_{\Gamma}=0\right\}
$$

Using standard notations, when $\mu$ is constant we have $\mathbf{X}_{2}=\mathbb{H}_{0}(\operatorname{curl}, \Omega) \cap \mathbb{H}(\operatorname{div}, \Omega)$. We introduce a new bilinear form $a_{2} \in \mathcal{L}\left(\mathbf{X}_{2} \times \mathbf{X}_{2} ; \mathbb{R}\right)$ such that for all $\mathbf{b}$, $\mathbf{b}^{\prime}$ in $\mathbf{X}_{2}$ we have

$$
a_{2}\left(\mathbf{b}, \mathbf{b}^{\prime}\right)=\left(\frac{1}{\tilde{\sigma}} \nabla \times \mathbf{b}, \nabla \times \mathbf{b}^{\prime}\right)_{\Omega}+\left(\frac{1}{\mu^{2} \tilde{\sigma}} \nabla \cdot(\mu \mathbf{b}), \nabla \cdot\left(\mu \mathbf{b}^{\prime}\right)\right)_{\Omega}-\left(\mathbf{u} \times(\mu \mathbf{b}), \nabla \times \mathbf{b}^{\prime}\right)_{\Omega_{c}}
$$

We have the following coercivity property.

Lemma 2.4. Under the assumptions of Lemma 2.1, there are $\alpha>0$ and $\gamma \geq 0$ such that

$$
\forall \mathbf{b} \in \mathbf{X}_{2}, \quad a_{2}(\mathbf{b}, \mathbf{b})+\gamma\|\mathbf{b}\|_{0, \Omega}^{2} \geq \alpha\|\mathbf{b}\|_{\mathbf{X}_{2}}^{2}
$$

Proof. Use the same arguments as those in Lemma 2.1.

Lemma 2.5. If $\|\mathbf{u}\|_{0, \infty, \Omega_{c}}$ is small enough, $\mu$ is constant, and $\Omega$ is simply connected, then $a_{2}$ is strongly coercive in $\mathbf{X}_{2}$. 
Proof. This result is a consequence of the inequality

$$
\forall \mathbf{b} \in \mathbf{X}_{2}, \quad\|\nabla \times \mathbf{b}\|_{0, \Omega}^{2}+\|\nabla \cdot \mathbf{b}\|_{0, \Omega}^{2} \geq c\|\mathbf{b}\|_{\mathbf{X}_{2}}^{2} .
$$

See e.g. [12].

Remark 2.3. If, in addition to the hypotheses of Lemma $2.5, \Gamma$ is piecewise smooth with no reentrant corners, then the norm of $\mathbf{X}_{2}$ is equivalent to that of $\mathbb{H}^{1}(\Omega)$ (see [12, p. 44]). In astrophysical problems, $\Gamma$ can easily be chosen to satisfy this hypothesis.

Now we consider the following problem: For $j \in L^{2}\left(0, T ; L^{2}(\Omega)\right)$ and $\mathbf{H}_{0} \in \mathbf{L}$,

$$
\left\{\begin{array}{l}
\text { Find } \mathbf{H} \in W\left(0, T ; \mathbf{X}_{2}\right) \text { and } E \in \mathcal{D}^{\prime}(0, T ; M) \text { s.t., } \forall \mathbf{b} \in \mathbf{X}_{2}, \quad \forall e \in M \\
\left(\mu \partial_{t} \mathbf{H}, \mathbf{b}\right)_{\Omega}+a_{2}(\mathbf{H}, \mathbf{b})+(E, \nabla \times \mathbf{b})_{\Omega_{v}}=\left(\frac{1}{\sigma} j, \nabla \times \mathbf{b}\right)_{\Omega_{c}} \text { in } \mathcal{D}^{\prime}(0, T) \\
(\nabla \times \mathbf{H}, e)_{\Omega_{v}}=0 \quad \text { in } L^{2}(0, T) .
\end{array}\right.
$$

Theorem 2.3. Under the hypotheses of Lemma 2.4, problem (2.7) is wellposed and its solution is also solution to problem (2.2).

Proof. (1) The wellposedness is a simple consequence of the coercivity property stated in 2.4, Lions' theorem, and the fact that the linear mapping $\mathbf{X}_{2} \ni \mathbf{b} \longmapsto \nabla \times \mathbf{b} \in M$ is surjective.

(2) Let us show that the couple $(\mathbf{H}, E)$ is solution to problem (2.2). Let $1_{\Omega_{c}}$ be the characteristic function of $\Omega_{c}$. In the sense of distributions, $\mathbf{H}$ and $E$ satisfy the following PDE's

$$
\begin{cases}\mu \partial_{t} \mathbf{H}+\nabla \times\left(\frac{1}{\tilde{\sigma}} \nabla \times \mathbf{H}\right)-\mu \nabla\left(\frac{1}{\mu^{2} \tilde{\sigma}} \nabla \cdot(\mu \mathbf{H})\right)-\nabla \times\left(1_{\Omega_{c}} \mathbf{u} \times \mu \mathbf{H}\right) & \multicolumn{1}{c}{\quad+\nabla \times\left(1_{\Omega_{v}} E\right)=\nabla \times\left(\frac{1_{\Omega_{c}}}{\tilde{\sigma}} j\right) \quad \text { in } \Omega} \\ \nabla \times \mathbf{H}=0 & \text { in } \Omega_{v} \\ \nabla \cdot(\mu \mathbf{H})=0 & \text { on } \Gamma \\ \mathbf{H} \times \mathbf{n}=0 & \text { on } \Gamma \\ \mathbf{H}=H_{0} & \text { at } t=0 .\end{cases}
$$

Taking the divergence of the first equation, setting $\phi=\frac{1}{\mu^{2} \tilde{\sigma}} \nabla \cdot(\mu \mathbf{H})$, and using the fact that $\nabla \cdot \mathbf{H}_{0}=0$, we obtain

$$
\begin{cases}\mu^{2} \tilde{\sigma} \partial_{t} \phi-\nabla \cdot(\mu \nabla \phi)=0 & \text { in } \Omega \\ \phi=0 & \text { on } \Gamma \\ \phi=0 & \text { at } t=0 .\end{cases}
$$

Hence, $\phi$ is zero for all times, that is, $\nabla \cdot(\mu \mathbf{H})=0$. Owing to this property and the fact that $\nabla \times \mathbf{H}$ is zero in $\Omega_{v}$, it is clear that $\mathbf{H}$ is solution to problem (2.2).

Remark 2.4. We emphasize once more that, contrary to what is sometimes claimed by some authors, the equation $\nabla \cdot(\mu \mathbf{H})=0$ is not part of the original system (2.1). In other words, the constraint $\nabla \cdot(\mu \mathbf{H})=0$ needs not be enforced for (2.1) to be wellposed. This equation is just an a posteriori consequence of (2.1). Nevertheless, it is standard to incorporate this equation in stabilized formulations to have an a priori control on the divergence of $\mu \mathbf{H}$. For instance we refer to [22] or [9] where this type of stabilization is used in conjunction with standard Lagrange finite elements to solve MHD problems in conducting mediums with constant properties. Another possibility to enforce the additional constraint weakly consists in introducing a dummy Lagrange multiplier (see e.g. $[8])$. 
Remark 2.5. Of course, if $\mu$ is discontinuous across $\Sigma$, we only have $\mathbf{H} \in \mathbb{H}_{0}(\operatorname{curl}, \Omega)$ and $\mu \mathbf{H} \in \mathbb{H}(\operatorname{div}, \Omega)$. Hence, one should not expect $\mathbf{H}$ to be in $\mathbb{H}^{1}(\Omega)$ locally in the neighborhood of $\Sigma$, for $\mathbf{H} \cdot \mathbf{n}$ must be discontinuous across $\Sigma$. As a result, formulation (2.7) is not suitable when using continuous Lagrange finite elements if $\mu$ is discontinuous.

\section{Finite Element Discretization}

In this section we consider finite element discretization of the three formulations considered above. We shall denote by $\left(\mathcal{T}_{h}\right)_{h>0}$ a family of regular meshes of $\Omega$.

\subsection{The basic formulation}

We build first an approximate solution for problem (2.2). Let us introduce $\mathbf{X}_{1 h} \subset \mathbf{X}_{1}$ and $M_{h} \subset M$, two finite dimensional, finite element spaces based on the mesh $\mathcal{T}_{h}$. Denoting by $\mathcal{I}_{h}$ a suitable interpolation operator that maps $\mathbf{X}_{1}$ onto $\mathbf{X}_{1 h}$, we build an approximate solution as follows.

$$
\left\{\begin{array}{l}
\text { Find } \mathbf{H}_{h} \in \mathcal{C}^{1}\left(0, T ; \mathbf{X}_{1 h}\right) \text { and } E_{h} \in \mathcal{C}^{0}\left(0, T ; M_{h}\right) \text { s.t., } \forall \mathbf{b}_{h} \in \mathbf{X}_{1 h}, \forall e_{h} \in M_{h} \\
\left(\mu d_{t} \mathbf{H}_{h}, \mathbf{b}_{h}\right)_{\Omega}+a_{1}\left(\mathbf{H}_{h}, \mathbf{b}_{h}\right)+\left(E_{h}, \nabla \times \mathbf{b}_{h}\right)_{\Omega_{v}}=\left(\frac{1}{\sigma} j, \nabla \times \mathbf{b}_{h}\right)_{\Omega_{c}} \\
\left(\nabla \times \mathbf{H}_{h}, e_{h}\right)_{\Omega_{v}}=0 \\
\left.\mathbf{H}\right|_{t=0}=\mathcal{I}_{h} \mathbf{H}_{0} .
\end{array}\right.
$$

The convergence analysis for this type of problem is quite standard, we refer to [2] or [12] for details. The critical step consists in building an adequate interpolate of the continuous solution. Generally, this is done by looking for $I_{h} \mathbf{H} \in \mathbf{X}_{1 h}$ and $J_{h} E \in M_{h}$ so that

$$
\left\{\begin{array}{l}
\tilde{a}_{1}\left(I_{h} \mathbf{H}, \mathbf{b}_{h}\right)+\left(J_{h} E, \nabla \times \mathbf{b}_{h}\right)_{\Omega_{v}}=\tilde{a}_{1}\left(\mathbf{H}, \mathbf{b}_{h}\right)+\left(E, \nabla \times \mathbf{b}_{h}\right)_{\Omega_{v}} \quad \forall \mathbf{b}_{h} \in \mathbf{X}_{1 h} \\
\left(\nabla \times I_{h} \mathbf{H}_{h}, e_{h}\right)_{\Omega_{v}}=0 \quad \forall e_{h} \in M_{h}
\end{array}\right.
$$

with $\tilde{a}_{1}\left(\mathbf{b}, \mathbf{b}^{\prime}\right)=\gamma\left(\mathbf{b}, \mathbf{b}^{\prime}\right)_{0, \Omega}+a_{1}\left(\mathbf{b}, \mathbf{b}^{\prime}\right)$, where $\gamma$ is chosen sufficiently large for $\tilde{a}_{1}$ to be coercive in $\mathbf{V}$.

Let us set $\mathbf{V}_{h}=\left\{\mathbf{b}_{h} \in \mathbf{X}_{1 h},\left(\nabla \times \mathbf{b}_{h}, e_{h}\right)_{\Omega_{v}}=0, \forall e_{h} \in M_{h}\right\}$. Then, necessary and sufficient conditions for problem (3.2) to be wellposed are as follows

$$
\begin{gathered}
\exists \alpha_{h}>0, \forall \mathbf{b}_{h} \in \mathbf{V}_{h}, \quad \sup _{\mathbf{b}_{h}^{\prime} \in \mathbf{V}_{h}} \frac{\tilde{a}_{1}\left(\mathbf{b}_{h}, \mathbf{b}_{h}^{\prime}\right)}{\left\|\mathbf{b}_{h}^{\prime}\right\|_{\mathbf{X}_{1}}} \geq \alpha_{h}\left\|\mathbf{b}_{h}\right\| \mathbf{x}_{1}, \\
\exists \beta_{h}>0, \forall \mathbf{e}_{h} \in \mathbf{M}_{h}, \quad \sup _{\mathbf{b}_{h} \in \mathbf{X}_{1 h}} \frac{\left(\nabla \times \mathbf{b}_{h}, e_{h}\right)_{\Omega_{v}}}{\left\|\mathbf{b}_{h}\right\|_{\mathbf{X}_{1}}} \geq \beta_{h}\left\|e_{h}\right\|_{M} .
\end{gathered}
$$

We shall see below that it is possible to define $\mathbf{X}_{1 h}$ and $M_{h}$ so that (3.4) is satisfied uniformly with respect to $h$. Now let us turn our attention to (3.3). If for a moment we assume that $\mathbf{u}$ is zero, then $\tilde{a}_{1}$ is symmetric and positive. In this case, (3.3) is equivalent to assuming coercivity for $\tilde{a}_{1}$. Unfortunately, though $\tilde{a}_{1}$ is coercive on $\mathbf{V}$, it is generally not coercive on $\mathbf{V}_{h}$ unless $\mathbf{V}_{h} \subset \mathbf{V}$. It is at this very point that approaches based on standard Lagrange finite elements differ from approaches based on edge finite elements. For Lagrange elements $\mathbf{V}_{h}$ is generally not a subset of $\mathbf{V}$, whereas with edge elements it is possible to build $V_{h}$ so that $\mathbf{V}_{h} \subset \mathbf{V}$.

The provisional conclusion at this point is that formulation (2.2) is a priori not suitable for an approximation with Lagrange finite elements. However, it is shown in Section 4 that by using $\mathbb{P}_{2}$ finite elements for $\mathbf{X}_{1 h}$ and $\mathbb{P}_{1}$ finite elements for $M_{h}$, the solution to (3.1) does converge. We observe $h^{2}$ rate on $E$ in the $L^{2}$-norm, $h^{3 / 2}$ rate on $\mathbf{H}$ in the $\mathbb{L}^{2}$-norm, and $h^{1 / 2}$ rate in the $\mathbb{H}^{1}$-norm. Though, we have not proved these results yet. 


\subsection{The partially stabilized formulation}

We now turn our attention to the partially stabilized formulation (2.6).

Let us introduce $\mathbf{X}_{1 h}$ and $M_{h}$ two internal finite element approximations of $\mathbf{X}_{1}$ and $M$ based on the mesh $\mathcal{T}_{h}$. It is assumed hereafter that the following properties hold. There exist $c>0$ and $k>1$ such that for all $0 \leq r \leq k$

$$
\begin{gathered}
\inf _{\mathbf{b}_{h} \in \mathbf{X}_{1 h}}\left(\left\|\mathbf{b}-\mathbf{b}_{h}\right\|_{0, \Omega}+h\left\|\mathbf{b}-\mathbf{b}_{h}\right\|_{\mathbf{X}_{1}}\right) \leq c h^{r+1}\|\mathbf{b}\|_{r+1, \Omega}, \quad \forall \mathbf{b} \in \mathbf{H}^{r+1}(\Omega) \cap \mathbf{X}_{2}, \\
\inf _{e_{h} \in M_{h}}\left\|e-e_{h}\right\|_{0, \Omega_{v}} \leq c h^{r}\|e\|_{r, \Omega_{v}} . \quad \forall e \in H^{r}\left(\Omega_{v}\right) \cap M .
\end{gathered}
$$

We assume also that there is $\beta>0$, independent of $h$, so that

$$
\forall \mathbf{e}_{h} \in \mathbf{M}_{h}, \quad \sup _{\mathbf{b}_{h} \in \mathbf{X}_{1 h}} \frac{\left(\nabla \times \mathbf{b}_{h}, e_{h}\right)_{\Omega_{v}}}{\left\|\mathbf{b}_{h}\right\| \mathbf{X}_{1}} \geq \beta\left\|e_{h}\right\|_{M}
$$

We assume that there exists an interpolation operator $\mathcal{I}_{h}: \mathbf{X}_{2} \longrightarrow \mathbf{X}_{2 h}$, so that $\left\|\mathbf{H}_{0}-\mathcal{I}_{h} \mathbf{H}_{0}\right\|_{0, \Omega} \leq c h^{k+1}\left\|\mathbf{H}_{0}\right\|_{k+1, \Omega}$. We build an approximate solution as follows.

$$
\left\{\begin{array}{l}
\text { Find } \mathbf{H}_{h} \in \mathcal{C}^{1}\left(0, T ; \mathbf{X}_{1 h}\right) \text { and } E_{h} \in \mathcal{C}^{0}\left(0, T ; M_{h}\right) \text { s.t., } \forall \mathbf{b}_{h} \in \mathbf{X}_{1 h}, \forall e_{h} \in M_{h} \\
\left(\mu d_{t} \mathbf{H}_{h}, \mathbf{b}_{h}\right)_{\Omega}+a_{1, s}\left(\mathbf{H}_{h}, \mathbf{b}_{h}\right)+\left(E_{h}, \nabla \times \mathbf{b}_{h}\right)_{\Omega_{v}}=\left(\frac{1}{\sigma} j, \nabla \times \mathbf{b}_{h}\right)_{\Omega_{c}} \\
\left(\nabla \times \mathbf{H}_{h}, e_{h}\right)_{\Omega_{v}}=0 \\
\mathbf{H}_{h \mid t=0}=\mathcal{I}_{h} \mathbf{H}_{0} .
\end{array}\right.
$$

Theorem 3.1. Under the hypotheses of Lemma 2.3, if the solution to (2.2) is such that $\mathbf{H} \in \mathcal{C}^{1}\left([0, \infty] ; \mathbf{H}^{k+1}(\Omega)\right)$ and $E \in \mathcal{C}^{0}\left([0, \infty] ; H^{k}(\Omega)\right)$, then the solution to (3.6) is s.t. for all $t>0$

$$
\left\|\mathbf{H}(t)-\mathbf{H}_{h}(t)\right\|_{0, \Omega}+\left[\frac{1}{t} \int_{0}^{t}\left\|\mathbf{H}-\mathbf{H}_{h}\right\|_{\mathbf{X}_{1}}^{2}+\left\|E-E_{h}\right\|_{0, \Omega_{v}}^{2}\right]^{1 / 2} \leq c h^{k} c(t, \mathbf{H}, E) .
$$

Proof. The arguments are standard.

Remark 3.1. Note that we cannot expect from (3.6) to obtain extra convergence on $\mathbf{H}_{h}$ in the $\mathbb{L}^{2}(\Omega)$-norm through the Nitsche-Aubin trick, for the dual problem associated with the bilinear form $a_{1 s}$ has no regularization property.

\subsection{The fully stabilized formulation}

We now turn our attention to the fully stabilized formulation (2.7) and we adopt all the hypotheses that pertain to it.

Let us introduce $\mathbf{X}_{2 h}$ and $M_{h}$ two internal finite element approximations of $\mathbf{X}_{2}$ and $M$ based on the mesh $\mathcal{T}_{h}$. We assume that the following approximation properties hold. There exist $c>0$ and $k>1$ such that for all $0 \leq r \leq k$

$$
\begin{gathered}
\inf _{\mathbf{b}_{h} \in \mathbf{X}_{2 h}}\left(\left\|\mathbf{b}-\mathbf{b}_{h}\right\|_{0, \Omega}+h\left\|\mathbf{b}-\mathbf{b}_{h}\right\|_{\mathbf{X}_{2}}\right) \leq c h^{r+1}\|\mathbf{b}\|_{r+1, \Omega}, \quad \forall \mathbf{b} \in \mathbf{H}^{r+1}(\Omega) \cap \mathbf{X}_{2}, \\
\inf _{e_{h} \in M_{h}}\left\|e-e_{h}\right\|_{0, \Omega_{v}} \leq c h^{r}\|e\|_{r, \Omega_{v}} . \quad \forall e \in H^{r}\left(\Omega_{v}\right) \cap M .
\end{gathered}
$$


Furthermore, we assume that $\mathbf{u}$ is small enough for $a_{2}$ to be strongly coercive in $\mathbf{X}_{2}$ according to Lemma 2.5. This hypothesis is not essential, but we use it for it yields uniform boundedness in time. We assume also that the following inf-sup condition holds: there is $\beta>0$, independent of $h$, so that

$$
\forall \mathbf{e}_{h} \in \mathbf{M}_{h}, \quad \sup _{\mathbf{b}_{h} \in \mathbf{X}_{2 h}} \frac{\left(\nabla \times \mathbf{b}_{h}, e_{h}\right)_{\Omega_{v}}}{\left\|\mathbf{b}_{h}\right\|_{\mathbf{X}_{2}}} \geq \beta\left\|e_{h}\right\|_{M}
$$

We denote by $\mathcal{I}_{h}: \mathbf{X}_{2} \longrightarrow \mathbf{X}_{2 h}$ an interpolation operator s.t. $\left\|\mathbf{H}_{0}-\mathcal{I}_{h} \mathbf{H}_{0}\right\|_{0, \Omega} \leq c h^{k+1}\left\|\mathbf{H}_{0}\right\|_{k+1, \Omega}$. We build an approximate solution to $(2.7)$ as follows.

$$
\left\{\begin{array}{l}
\text { Find } \mathbf{H}_{h} \in \mathcal{C}^{1}\left(0, T ; \mathbf{X}_{2 h}\right) \text { and } E_{h} \in \mathcal{C}^{0}\left(0, T ; M_{h}\right) \text { s.t., } \forall \mathbf{b}_{h} \in \mathbf{X}_{2 h}, \forall e_{h} \in M_{h} \\
\left(\mu d_{t} \mathbf{H}_{h}, \mathbf{b}_{h}\right)_{\Omega}+a_{2}\left(\mathbf{H}_{h}, \mathbf{b}_{h}\right)+\left(E_{h}, \nabla \times \mathbf{b}_{h}\right)_{\Omega_{v}}=\left(\frac{1}{\sigma} j, \nabla \times \mathbf{b}_{h}\right)_{\Omega_{c}} \\
\left(\nabla \times \mathbf{H}_{h}, e_{h}\right)_{\Omega_{v}}=0 \\
\mathbf{H}_{h \mid t=0}=\mathcal{I}_{h} \mathbf{H}_{0} .
\end{array}\right.
$$

Theorem 3.2. Under the hypotheses of Lemma 2.5, if the solution to (2.2) is such that $\mathbf{H} \in \mathcal{C}^{1}\left([0, \infty] ; \mathbf{H}^{k+1}(\Omega)\right)$ and $E \in \mathcal{C}^{0}\left([0, \infty] ; H^{k}(\Omega)\right)$, then the solution to (3.8) satisfies the following error estimates

$$
\begin{aligned}
& \forall t>0, \quad\left\|\mathbf{H}(t)-\mathbf{H}_{h}(t)\right\|_{0, \Omega} \leq\left\|\mathbf{H}_{0}-\mathcal{I}_{h} \mathbf{H}_{0}\right\|_{0, \Omega} \mathrm{e}^{-c_{1} t}+c_{2} h^{k} c(\mathbf{H}, E), \\
& {\left[\frac{1}{t} \int_{0}^{t}\left\|\mathbf{H}-\mathbf{H}_{h}\right\|_{\mathbf{X}_{2}}^{2}+\left\|E-E_{h}\right\|_{0, \Omega_{v}}^{2}\right]^{1 / 2} \leq c_{3} h^{k}\left(1+\frac{1}{\sqrt{t}}\right) c(\mathbf{H}, E), }
\end{aligned}
$$

with $c(\mathbf{H}, E)=\|\mathbf{H}\|_{\mathcal{C}^{1}\left([0, \infty] ; \mathbf{H}^{k+1}(\Omega)\right)}+\|E\|_{\mathcal{C}^{1}\left([0, \infty] ; H^{k}(\Omega)\right)}$.

Proof. The arguments are standard.

Contrary to formulation (3.6), extra convergence on $\mathbf{H}_{h}$ in the $\mathbb{L}^{2}(\Omega)$-norm can be achieved if the domain is such that some regularization properties hold. More precisely, setting $\tilde{a}_{2}(\mathbf{b}, \boldsymbol{\phi})=(\mathbf{b}, \boldsymbol{\phi})_{0, \Omega}+a_{2}(\mathbf{b}, \boldsymbol{\phi})$, let us assume that there exists $0<r \leq 1$ such that for all $\boldsymbol{\psi}$ in $\mathbb{L}^{2}(\Omega)$, there are $\phi \in \mathbf{H}^{1+r}(\Omega) \cap \mathbf{X}_{2}$ and $\alpha \in H^{r}(\Omega) \cap M$ satisfying

$$
\left(H_{r}\right) \quad \begin{cases}\tilde{a}_{2}(\mathbf{b}, \boldsymbol{\phi})+(\alpha, \nabla \times \mathbf{b})_{\Omega_{v}}=(\boldsymbol{\psi}, \mathbf{b})_{0, \Omega} & \forall \mathbf{b} \in \mathbf{X}_{2} \\ (\nabla \times \phi, e)_{\Omega_{v}}=0 & \forall e \in M\end{cases}
$$

and $\|\phi\|_{1+r, \Omega}+\|\alpha\|_{r, \Omega_{v}} \leq c\|\boldsymbol{\psi}\|_{0, \Omega}$. Then, we have the following result.

Proposition 3.1. Under the above regularization hypothesis, $\left(H_{r}\right)$, and the hypotheses of Theorem 3.2 we have

$$
\forall t>0, \quad\left\|\mathbf{H}(t)-\mathbf{H}_{h}(t)\right\|_{0, \Omega} \leq c h^{k+r} c(\mathbf{H}, E) .
$$

Proof. Use the standard duality argument of Nitsche-Aubin to build suitable interpolates of $\mathbf{H}$ and $E$.

Remark 3.2. Note that the error estimates in Theorem 3.2 hold for arbitrarily large times. This property is a consequence of the strong coercivity of $a_{2}$ in $\mathbf{X}_{2}$ (cf. Lem. 2.5). If $a_{2}$ is only coercive according to Lemma 2.4, then the estimates in Theorem 3.2 and Proposition 3.1 still hold with constants that depend on $t$. 


\subsection{Fully discrete formulation}

To obtain a fully discretized problem, we use the second order backward difference. Then, for $i=1$ or 2 , the fully discrete system reads

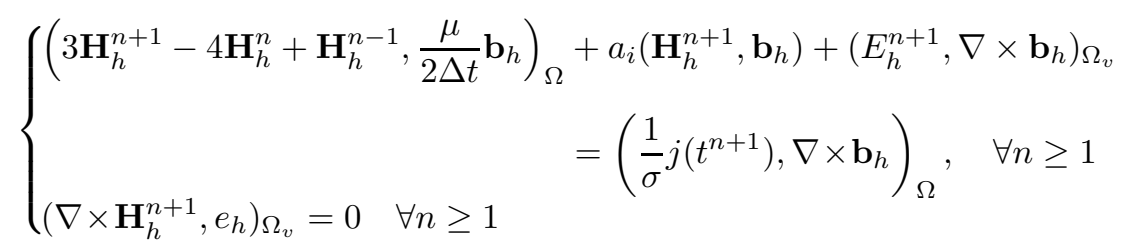

assuming a suitable initialization for $\mathbf{H}_{h}^{0}$ and using the implicit Euler scheme to compute $\mathbf{H}_{h}^{1}$.

For a given time $t \geq 0$, a Hilbert space $G$, and a sequence in $\mathrm{G}, \alpha^{0}, \alpha^{1}, \ldots$, we introduce the following discrete norms

$$
\|\alpha\|_{l^{2}(G)}=\left(\frac{\Delta t}{t} \sum_{n=0}^{[t / \Delta t]}\left\|\alpha^{n}\right\|_{G}^{2}\right)^{1 / 2}, \quad\|\alpha\|_{l^{\infty}(G)}=\max _{0 \leq n \leq[t / \Delta t]}\left\|\alpha^{n}\right\|_{G},
$$

where $[t / \Delta t]$ denotes the integer part of $t / \Delta t$. In the case of the stabilized formulation, it is an easy matter to derive the following result.

Proposition 3.2. If $\left\|\mathbf{H}_{h}^{0}-\mathbf{H}_{0}\right\|_{0, \Omega} \leq c h^{k+1}$, if the solution to (2.2) is sufficiently smooth in time, and under the hypotheses of Theorem 3.1 or 3.2, we have for all $t \geq 0$

$$
\left\|\mathbf{H}_{h}-\mathbf{H}\right\|_{l^{2}\left(\mathbf{X}_{i}\right)}+\left\|E-E_{h}\right\|_{l^{2}(M)} \leq c\left(\Delta t^{2}+h^{k}\right)
$$

and under the hypotheses of Proposition 3.1, we have

$$
\left\|\mathbf{H}_{h}-\mathbf{H}\right\|_{l^{\infty}\left(\mathbb{L}^{2}(\Omega)\right)} \leq c\left(\Delta t^{2}+h^{k+r}\right) .
$$

\subsection{Inf-sup stable Lagrange finite elements}

We shall prove in this section that (3.5) and (3.7) hold for some mixed Lagrange finite element.

\subsubsection{The mini finite element}

Let us consider first the so-called mini element with $\mathbb{P}_{1}$-bubble/ $\mathbb{P}_{1}$ approximation for $\mathbf{H}$ and $E$. For simplicity, let us assume that $\Omega$ is a polygon and that $\mathcal{T}_{h}$ is composed of triangles. Furthermore, we assume that the interface of $\Omega_{v}$ and $\Omega_{c}$, i.e. $\Sigma$, is covered exactly with edges of triangles from the mesh.

Let $\widehat{K}$ be the reference simplex and $\widehat{b}$ be a bubble function on $\widehat{K}$, i.e. a function s.t.

$$
\widehat{b} \in H_{0}^{1}(\widehat{K}), \quad 0 \leq \widehat{b} \leq 1, \quad \widehat{b}(\widehat{C})=1,
$$

where $\widehat{C}$ is the barycenter of $\widehat{K}$. For each $K$ in $\mathcal{T}_{h}$, we denote be $T_{K}: \widehat{K} \longrightarrow K$ the affine transformation that maps $\widehat{K}$ into $K$. Then, we define $\widehat{\mathbf{P}}=\left(\mathbb{P}_{1}(\widehat{K}) \oplus \operatorname{span}(\widehat{b})\right)^{2}$, and we set

$$
\left\{\begin{array}{l}
\mathbf{X}_{2 h}=\left\{\mathbf{b}_{h} \in \mathbf{X}_{2} \cap \mathcal{C}^{0}\left(\bar{\Omega}_{v}\right), \mathbf{b}_{h} \circ T_{K} \in \widehat{\mathbf{P}}, \forall K \in \mathcal{T}_{h}\right\}, \\
M_{h}=\left\{e_{h} \in M \cap \mathcal{C}^{0}\left(\bar{\Omega}_{v}\right), e_{h} \circ T_{K} \in \mathbb{P}_{1}, \forall K \in \mathcal{T}_{h, v}\right\}
\end{array}\right.
$$

where $\mathcal{T}_{h, v}$ denotes the subset of the elements of $\mathcal{T}_{h}$ covering $\Omega_{v}$. 
The stability of this approximation is established in the following lemma.

Lemma 3.1. Under the above assumptions, there exists $\beta>0$ such that

$$
\sup _{\mathbf{b}_{h} \in \mathbf{X}_{2 h}} \frac{\left(\nabla \times \mathbf{b}_{h}, e_{h}\right)_{\Omega_{v}}}{\left\|\mathbf{b}_{h}\right\| \mathbf{X}_{2}} \geq \beta\left\|e_{h}\right\|_{M}, \quad \forall e_{h} \in M_{h}
$$

Proof. (1) Let $\mathbf{a}_{h}^{0}$ be a continuous vector-valued function on $\partial \Omega_{v}=\Sigma \cup \Gamma_{v}$ that is zero on $\Gamma_{v}$, piecewise $\mathbb{P}_{1}$ on the trace of the mesh $\mathcal{T}_{h}$ on $\partial \Omega_{v}$, and such that $\int_{\partial \Omega_{v}} \mathbf{a}_{h}^{0} \cdot \mathrm{d} \mathbf{l}=1$ and its norm in $\mathbf{H}^{1 / 2}\left(\partial \Omega_{v}\right)$ is bounded uniformly with respect to $h$. It is always possible to find such functions.

(2) For an arbitrary function $e_{h}$ in $M_{h}$, let us define $\mathbf{a}_{h}\left(e_{h}\right)=\mathbf{a}_{h}^{0} \int_{\Omega_{v}} e_{h}$. It is clear that

$$
\int_{\partial \Omega_{v}} \mathbf{a}_{h}\left(e_{h}\right) \cdot \mathrm{d} \mathbf{l}=\int_{\Omega_{v}} e_{h}, \quad \text { and } \quad\left\|\mathbf{a}_{h}\left(e_{h}\right)\right\|_{1 / 2, \partial \Omega_{v}} \leq\left\|\mathbf{a}_{h}^{0}\right\|_{1 / 2, \partial \Omega_{v}} \operatorname{meas}\left(\Omega_{v}\right)^{1 / 2}\left\|e_{h}\right\|_{0, \Omega_{v}} .
$$

Owing to the lifting Lemma 3.2, there is $\mathbf{b}\left(e_{h}\right)$ such that

$$
\nabla \times \mathbf{b}\left(e_{h}\right)=e_{h},\left.\quad \mathbf{b}\left(e_{h}\right)\right|_{\partial \Omega_{v}}=\mathbf{a}_{h}\left(e_{h}\right), \quad \text { and } \quad\left\|\mathbf{b}\left(e_{h}\right)\right\|_{1, \Omega_{v}} \leq c\left\|e_{h}\right\|_{0, \Omega_{v}} .
$$

Since $\Omega_{v}$ is Lipschitz, we can extend $\mathbf{b}$ in $\mathbb{H}^{1}(\Omega)$ (see e.g. [18, pp. 65-81]). Let $\widetilde{\mathbf{b}}$ be one of these extensions, and let $\mathcal{C}_{h}(\widetilde{\mathbf{b}})$ be the Clément interpolate of $\widetilde{\mathbf{b}}$ such that $\left.\mathcal{C}_{h}(\widetilde{\mathbf{b}})\right|_{\partial \Omega_{v}}=\left.\widetilde{\mathbf{b}}\right|_{\partial \Omega_{v}}$ (see [5]). This is possible, for the trace of $\mathbf{b}$ on $\partial \Omega_{v}$ is exactly the trace of an element in $\mathbf{X}_{2 h}$. This interpolate is such that

$$
\left\|\mathcal{C}_{h}(\widetilde{\mathbf{b}})\right\|_{1, \Omega} \leq c\|\widetilde{\mathbf{b}}\|_{1, \Omega} \leq c^{\prime}\left\|e_{h}\right\|_{0, \Omega_{v}} .
$$

(3) Now we choose the bubble function $\mathbf{B}_{h}(\widetilde{\mathbf{b}})$ so that

$$
\int_{T} \widetilde{\mathbf{b}}=\int_{T}\left(\mathcal{C}_{h}(\widetilde{\mathbf{b}})+\mathbf{B}_{h}(\widetilde{\mathbf{b}})\right), \quad T \in \mathcal{T}_{h}
$$

and we set $\boldsymbol{\Pi}_{h}(\widetilde{\mathbf{b}})=\mathcal{C}_{h}(\widetilde{\mathbf{b}})+\mathbf{B}_{h}(\widetilde{\mathbf{b}})$. From standard arguments, (see e.g. [2,12]), we infer

$$
\left\|\Pi_{h}(\widetilde{\mathbf{b}})\right\|_{1, \Omega} \leq c\|\widetilde{\mathbf{b}}\|_{1, \Omega} \leq c^{\prime}\left\|e_{h}\right\|_{M} .
$$

(4) From the definitions above, we deduce

$$
\begin{aligned}
0 & =\int_{\Omega_{v}}\left(\mathbf{b}-\boldsymbol{\Pi}_{h}(\widetilde{\mathbf{b}})\right) \cdot \nabla \times e_{h}, & & \text { since } \nabla \times e_{h} \text { is } \mathbb{P}_{0} \text { on each triangle } \\
& =\int_{\Omega_{v}} e_{h} \nabla \times\left(\mathbf{b}-\boldsymbol{\Pi}_{h}(\widetilde{\mathbf{b}})\right), & & \text { since } \mathbf{b}=\boldsymbol{\Pi}_{h}(\widetilde{\mathbf{b}}) \text { on } \Sigma \cup \Gamma_{v} .
\end{aligned}
$$

As a result

$$
\begin{aligned}
\sup _{\mathbf{b}_{h} \in \mathbf{X}_{2 h}} \frac{\left(\nabla \times \mathbf{b}_{h}, e_{h}\right)_{\Omega_{v}}}{\left\|\mathbf{b}_{h}\right\|_{\mathbf{X}_{2}}} & \geq \frac{\left(\nabla \times \boldsymbol{\Pi}_{h}(\widetilde{\mathbf{b}}), e_{h}\right)_{\Omega_{v}}}{\left\|\boldsymbol{\Pi}_{h}(\widetilde{\mathbf{b}})\right\|_{\mathbf{X}_{2}}}=\frac{\left(\nabla \times \mathbf{b}, e_{h}\right)_{\Omega_{v}}}{\left\|\boldsymbol{\Pi}_{h}(\widetilde{\mathbf{b}})\right\|_{\mathbf{X}_{2}}} \\
& =\frac{\left\|e_{h}\right\|_{M}^{2}}{\left\|\boldsymbol{\Pi}_{h}(\widetilde{\mathbf{b}})\right\|_{\mathbf{X}_{2}}} \geq c\left\|e_{h}\right\|_{M} .
\end{aligned}
$$

This completes the proof. 
Remark 3.3. Note that the key argument in the proof of Lemma 3.1 is to build a vector field $\mathbf{b}$ in $\mathbb{H}^{1}\left(\Omega_{v}\right)$ so that its trace on $\Sigma \cup \Gamma_{v}$ is the trace of an element in $\mathbf{X}_{2 h}$. This guarantees that integration by parts in $\left(\nabla \times\left(\mathbf{b}-\boldsymbol{\Pi}_{h}(\widetilde{\mathbf{b}})\right), e_{h}\right)_{\Omega_{v}}$ can be performed with no boundary integral left.

Lemma 3.2. Let $\mathcal{O}$ be a Lipschitz domain in $\mathbb{R}^{2}$. For all $\mathbf{a} \in \mathbf{H}^{1 / 2}(\partial \mathcal{O})$ and $e \in L^{2}(\mathcal{O})$ such that $\int_{\mathcal{O}} e=$ $\int_{\partial \mathcal{O}} \mathbf{a} \cdot \mathrm{d} \mathbf{l}$, there is $\mathbf{u} \in \mathbb{H}^{1}(\mathcal{O})$ so that $\nabla \times \mathbf{u}=e$ and $\left.\mathbf{u}\right|_{\partial \mathcal{O}}=\mathbf{a}$.

Proof. Let us denote $\mathbf{a}^{\star}=\left(a_{2},-a_{1}\right)$. From the theory of the divergence operator we know that there is $\mathbf{w} \in \mathbb{H}^{1}(\mathcal{O})$ so that $\nabla \cdot \mathbf{w}=e$ and $\left.\mathbf{w}\right|_{\partial \mathcal{O}}=\mathbf{a}^{\star}$. Now, setting $\mathbf{u}=\left(-w_{2}, w_{1}\right)$, it is clear that $\mathbf{u}$ satisfies the required conditions.

\subsubsection{The $\mathbb{P}_{2} / \mathbb{P}_{1}$ element}

We now turn our attention to the $\mathbb{P}_{2}-\mathbb{P}_{1}$ element which is known in the literature on the Stokes problem as the Taylor-Hood element (see [2]).

In addition to the hypotheses already made on the mesh and the domain for the mini element, we assume now that each triangle that has a nonempty intersection with $\partial \Omega_{v}$ has at most one edge on $\partial \Omega_{v}$.

We define the approximation spaces as follows:

$$
\left\{\begin{array}{l}
\mathbf{X}_{2 h}=\left\{\mathbf{b}_{h} \in \mathbf{X}_{2} \cap \mathcal{C}^{0}(\bar{\Omega}), \mathbf{b}_{h} \circ T_{K} \in\left(\mathbb{P}_{2}\right)^{2}, \forall K \in \mathcal{T}_{h}\right\}, \\
M_{h}=\left\{e_{h} \in M \cap \mathcal{C}^{0}\left(\bar{\Omega}_{v}\right), e_{h} \circ T_{K} \in \mathbb{P}_{1}, \forall K \in \mathcal{T}_{h, v}\right\}
\end{array}\right.
$$

Lemma 3.3. Under the above assumptions, there exists $\beta>0$ such that

$$
\sup _{\mathbf{b}_{h} \in \mathbf{X}_{2 h}} \frac{\left(\nabla \times \mathbf{b}_{h}, e_{h}\right)_{\Omega_{v}}}{\left\|\mathbf{b}_{h}\right\|_{\mathbf{X}_{2}}} \geq \beta\left\|e_{h}\right\|_{M}, \quad \forall e_{h} \in M_{h}
$$

Proof. We adapt an idea developed in [23] for proving the inf-sup condition for the Taylor-Hood element. Let $e_{h}$ be a nonzero element in $M_{h}$. Let $\widetilde{\mathbf{b}}$ be as in the proof of Lemma 3.1 and $\mathcal{C}_{h}(\widetilde{\mathbf{b}})$ be its Clément interpolate. We have

$$
\begin{aligned}
\sup _{\mathbf{v}_{h} \in \mathbf{X}_{2 h}} \frac{\left(\nabla \times \mathbf{b}_{h}, e_{h}\right)_{\Omega_{v}}}{\left\|\mathbf{b}_{h}\right\|_{\mathbf{X}_{2}}} & \geq \frac{\left(\nabla \times \mathcal{C}_{h}(\widetilde{\mathbf{b}}), e_{h}\right)_{\Omega_{v}}}{\left\|\mathcal{C}_{h}(\widetilde{\mathbf{b}})\right\|_{\mathbf{X}_{2}}} \geq c \frac{\left(\nabla \times \mathcal{C}_{h}(\widetilde{\mathbf{b}}), e_{h}\right)_{\Omega_{v}}}{\|\widetilde{\mathbf{b}}\|_{1, \Omega}} \\
& =c \frac{\left(\nabla \times \widetilde{\mathbf{b}}, e_{h}\right)_{\Omega_{v}}}{\|\widetilde{\mathbf{b}}\|_{1, \Omega}}+c \frac{\left(\nabla \times\left(\mathcal{C}_{h}(\widetilde{\mathbf{b}})-\widetilde{\mathbf{b}}\right), e_{h}\right)_{\Omega_{v}}}{\|\widetilde{\mathbf{b}}\|_{1, \Omega}} \\
& \geq c\left\|e_{h}\right\|_{0, \Omega}-c^{\prime}\left(\sum_{K \in \mathcal{T}_{h, v}} h_{K}^{2}\left|e_{h}\right|_{1, K}^{2}\right)^{1 / 2} .
\end{aligned}
$$

By using Lemma 3.4 below, we bound from below the negative term in the right hand side as follows

$$
\sup _{\mathbf{b}_{h} \in \mathbf{X}_{2 h}} \frac{\left(\nabla \times \mathbf{b}_{h}, e_{h}\right)_{\Omega_{v}}}{\left\|\mathbf{b}_{h}\right\| \mathbf{X}_{2}} \geq c_{1}\left\|e_{h}\right\|_{M}-c_{2} \sup _{\mathbf{b}_{h} \in \mathbf{X}_{2 h}} \frac{\left(\nabla \times \mathbf{b}_{h}, e_{h}\right)_{\Omega_{v}}}{\left\|\mathbf{b}_{h}\right\| \mathbf{X}_{2}}
$$

We derive easily the inf-sup inequality with the constant $c_{1} /\left(1+c_{2}\right)$.

Lemma 3.4. Under the hypotheses of Lemma 3.3, there is $c>0$ so that

$$
\sup _{\mathbf{b}_{h} \in \mathbf{X}_{2 h}} \frac{\left(\nabla \times \mathbf{b}_{h}, e_{h}\right)_{0, \Omega_{v}}}{\left\|\mathbf{b}_{h}\right\|_{\mathbf{X}_{2}}} \geq c\left(\sum_{K \in \mathcal{T}_{h, v}} h_{K}^{2}\left|e_{h}\right|_{1, K}^{2}\right)^{1 / 2} .
$$


Proof. Let $K$ be a triangle in $\mathcal{T}_{h}$. Let $m$ be a node in the middle of an edge of $K$ and let us denote by $a$ and $b$ the two extremities of this edge. We set $l=\|b-a\|$ the length of the edge and $\tau=(b-a) / l$ a unitary vector on the edge. Let $\tau^{\star}$ be the vector such that $\tau^{\star}=\left(-\tau_{2}, \tau_{1}\right)$ where $\tau_{1}$ and $\tau_{1}$ are the two Cartesian components of $\tau$.

We introduce $\mathbf{X}_{2 h, v} \subset \mathbf{X}_{2}$ s.t.

$$
\mathbf{X}_{2 h, v}=\left\{\mathbf{b}_{h} \in \mathbf{X}_{2 h}, \operatorname{supp}\left(\mathbf{b}_{h}\right) \subset \Omega_{v}\right\} .
$$

With all function $e_{h}$ in $M_{h}$ we associate a function $\mathbf{v}_{h} \in \mathbf{X}_{2 h, v}$ s.t. for all $K \in \mathcal{T}_{h, v}$,

$$
\begin{cases}\mathbf{v}_{h}(a)=0 & \text { if } a \text { is a vertex of } K, \\ \mathbf{v}_{h}(m)=-l^{2} \tau^{\star} \partial_{\tau} e_{h} & \text { if } m \text { is a node in the middle of an edge of } K \text { not on } \Gamma_{v} \\ \mathbf{v}_{h}(m)=0 & \text { if } m \text { is a node in the middle of an edge of } K \text { on } \Gamma_{v} .\end{cases}
$$

It is clear that $\mathbf{v}_{h} \in \mathbf{X}_{2 h, v}$ and

$$
\left\|\mathbf{v}_{h}\right\|_{1, K} \leq c h_{K}\left|e_{h}\right|_{1, K}, \quad \forall K \in \mathcal{T}_{h, v} .
$$

First, we remark that

$$
\begin{aligned}
\sup _{\mathbf{b}_{h} \in \mathbf{X}_{2 h}} \frac{\left(\nabla \times \mathbf{b}_{h}, e_{h}\right)_{\Omega_{v}}}{\left\|\mathbf{b}_{h}\right\|_{\mathbf{X}_{2}}} & \geq \sup _{\mathbf{b}_{h} \in \mathbf{X}_{2 h, v}} \frac{\left(\nabla \times \mathbf{b}_{h}, e_{h}\right)_{\Omega_{v}}}{\left\|\mathbf{b}_{h}\right\| \mathbf{X}_{2}}=\sup _{\mathbf{b}_{h} \in \mathbf{X}_{2 h, v}} \frac{\left(\mathbf{b}_{h}, \nabla \times e_{h}\right)_{\Omega_{v}}}{\left\|\mathbf{b}_{h}\right\|_{\mathbf{X}_{2}}} \\
& \geq \frac{\left.\left(\mathbf{v}_{h}, \nabla \times e_{h}\right)\right)_{\Omega_{v}}}{\left\|\mathbf{v}_{h}\right\| \mathbf{X}_{2}}
\end{aligned}
$$

Second, by using the quadrature formula

$$
\forall \phi \in \mathbb{P}_{2} \quad \int_{K} \phi(x) \mathrm{d} x=\frac{\operatorname{meas}(K)}{3} \sum_{m} \phi(m),
$$

where $m$ spans the set of the three nodes on the edges of $\mathrm{K}$, we infer

$$
\begin{aligned}
\left(\mathbf{v}_{h}, \nabla \times e_{h}\right)_{\Omega_{v}} & =\sum_{K \in \mathcal{T}_{h, v}} \int_{K} \mathbf{v}_{h} \cdot \nabla \times e_{h} \\
& =\sum_{K \in \mathcal{T}_{h, v}} \sum_{m \in K} \mathbf{v}_{h}(m) \cdot \nabla \times e_{h}(m) \frac{\operatorname{meas}(K)}{3} \\
& =\sum_{K \in \mathcal{T}_{h, v}} \sum_{m \in K}\left|\partial_{\tau} e_{h}(m)\right|^{2} l^{2} \frac{\operatorname{meas}(K)}{3} \geq c \sum_{K \in \mathcal{T}_{h, v}} h_{K}^{2}\left|e_{h}\right|_{1, K}^{2} .
\end{aligned}
$$

Note that the last inequality holds only if each triangle has at most one edge on $\partial \Omega_{v}$. The desired inequality follows easily.

\subsection{Lagrange FE vs. edge elements}

Though the goal of the present paper is to show that it is indeed possible to solve the set of equations (2.1) by means of standard continuous Lagrange finite elements, provided the problem is set in an adequate weak form, the reader must bear in mind that there are limitations to this approach.

First, it is not natural to enforce the continuity of $\mathbf{H} \cdot \mathbf{n}$ across the interface $\Sigma$, for this quantity may be discontinuous if $\mu$ is discontinuous; in this case, the stabilized bilinear form $a_{2}$ must not be used. Note, however, that no particular assumption on the continuity of $\mu$ is required for using bilinear form $a_{1 s}$. 
Second, the most important limitation is that if either both $\mu$ and $\Sigma$ are simultaneously not smooth or $\Omega$ is not convex, then it may happen that $\mathbf{H}$ has no more regularity in space than that of $\mathbf{X}_{1}$ or $\mathbf{X}_{2}$. In this circumstance, it is possible that the sequence $\left(\mathbf{H}_{h}\right)_{h>0}$ does not converge to $\mathbf{H}$. The reason for this is that if simultaneously $\mu$ is discontinuous and $\Sigma$ is not smooth or $\Omega$ is not convex, then $\mathbf{X}_{2} \cap \mathbb{H}^{1}(\Omega)$ is a closed subspace of $\mathbf{X}_{2}$ with a supplementary that is not zero (see Costabel [6]). Under these conditions, $\mathbf{X}_{2} \cap \mathbb{H}^{1}(\Omega)$ is not dense in $\mathbf{X}_{2}$; that is, there are elements in $\mathbf{X}_{2}$ that cannot be approximated by continuous Lagrange finite element functions, these functions being in $\mathbf{X}_{2} \cap \mathbb{H}^{1}(\Omega)$. Note, however, that in the astrophysical context, either $\mu$ or $\Sigma$ is smooth and $\Omega$ can easily be chosen to be convex, so that convergence is guaranteed even in the minimal regularity situation. Of course, none of the limitations mentioned above apply if edge finite element are used.

\section{Numerical RESUlts}

In this section we report on numerical tests performed on $\mathbb{P}_{2} / \mathbb{P}_{1}$ implementations of the three formulations described above using BDF2 as time stepping.

\subsection{Convergence tests}

We first test convergence in space using an analytic solution to $(2.1)$. We set $\Omega=]-2,+2\left[\right.$ and $\Omega_{c}=\{(x, y) \in$ $\left.\mathbb{R}^{2}, \sqrt{x^{2}+y^{2}}<1\right\}$. Taking $\sigma=1$ and $\mu=1$, we choose

$$
\left\{\begin{array}{l}
\mathbf{H}=\sin (t)(\sin (x) \exp (y),-\cos (x) \exp (y)) \\
E=-\cos (t) \sin (x) \exp (y)
\end{array}\right.
$$

with $\mathbf{u}=0$ and the source term

$$
j=\left\{\begin{array}{lll}
\sigma \cos (t) \sin (x) \exp (y) & \text { in } & \Omega_{c} \\
0 & \text { in } & \Omega_{v}
\end{array}\right.
$$

We use $\mathbb{P}_{2} / \mathbb{P}_{1}$ finite elements to approximate $\mathbf{H}$ and $E$ and BDF2 to march in time. The time step is chosen sufficiently small so that the space error dominates the time error for all the selected meshes. We make two series of convergence tests: one using the bilinear form $a_{1}$; the other using the stabilized bilinear form $a_{2}$.

The results are reported in Figure 2. From left to right, the results shown in the Figure 2 have been obtained by using formulations $(2.2,2.6)$, and $(2.7)$, respectively. The errors are measured by evaluating the difference between the approximate solution and the exact one and by using high order quadrature rules. On the first two graphs we show the maximum in time of the error on $E$ in the $L^{2}$-norm and that on $\mathbf{H}$ in the $\mathbb{L}^{2}$-norm and the $\mathbf{X}_{1}$-norm as functions of $h$. On the rightmost graph we show the $\mathbb{H}^{1}$-norm instead of the $\mathbf{X}_{1}$-norm; note that since $\Omega$ is convex, the norms of $\mathbf{X}_{2}$ and $\mathbb{H}^{1}(\Omega)$ are equivalent; hence, it is legitimate to use the $\mathbb{H}^{1}$-norm to measure the error.

As expected the method using the non-stabilized bilinear form $a_{1}$ converges but yields non-optimal convergence rates. For the error on $E$ in $L^{2}$-norm we observe a $\mathcal{O}\left(h^{2}\right)$ rate; for the error on $\mathbf{H}$ in $\mathbb{L}^{2}$-norm and in $\mathbf{X}_{1}$-norm we have $\mathcal{O}\left(h^{3 / 2}\right)$ and $\mathcal{O}\left(h^{1 / 2}\right)$ rates respectively. The method using the partially stabilized bilinear form $a_{1 s}$ is optimal on $E$ in the $L^{2}$-norm and on $\mathbf{H}$ in the $\mathbf{X}_{1}$-norm. As expected, it is sub-optimal on $\mathbf{H}$ in the $\mathbb{L}^{2}$-norm (see Rem. 3.1). For the method using the fully stabilized bilinear form $a_{2}$, we observe that the convergence rates match either the second order slope or the third order slope as expected from Theorem 3.2 and Proposition 3.1 .

To have some insight on the long-time behavior of the errors, we show in Figure 3 the time history of the error on $E$ in $L^{2}$-norm, the error on $\mathbf{H}$ in $\mathbf{X}_{1}$-norm or $\mathbb{H}^{1}$-norm (depending on the bilinear form used), and that on $\nabla \cdot \mathbf{H}$ in $L^{2}$-norm for $0 \leq t \leq 10$. From left to right, the results shown in the Figure 3 have been obtained by using formulations $(2.2,2.6)$, and $(2.7)$, respectively. It is clear that when using $a_{1}$, the $\mathbf{X}_{1}$-norm is not controlled. When using $a_{1 s}$ the $\mathbf{X}_{1}$-norm of $\mathbf{H}$ is controlled but its divergence is not controlled. As expected from the theory, the bilinear form $a_{2}$ yields satisfactory long-time behavior on $\mathbf{H}$ in the $\mathbb{H}^{1}$-norm. 

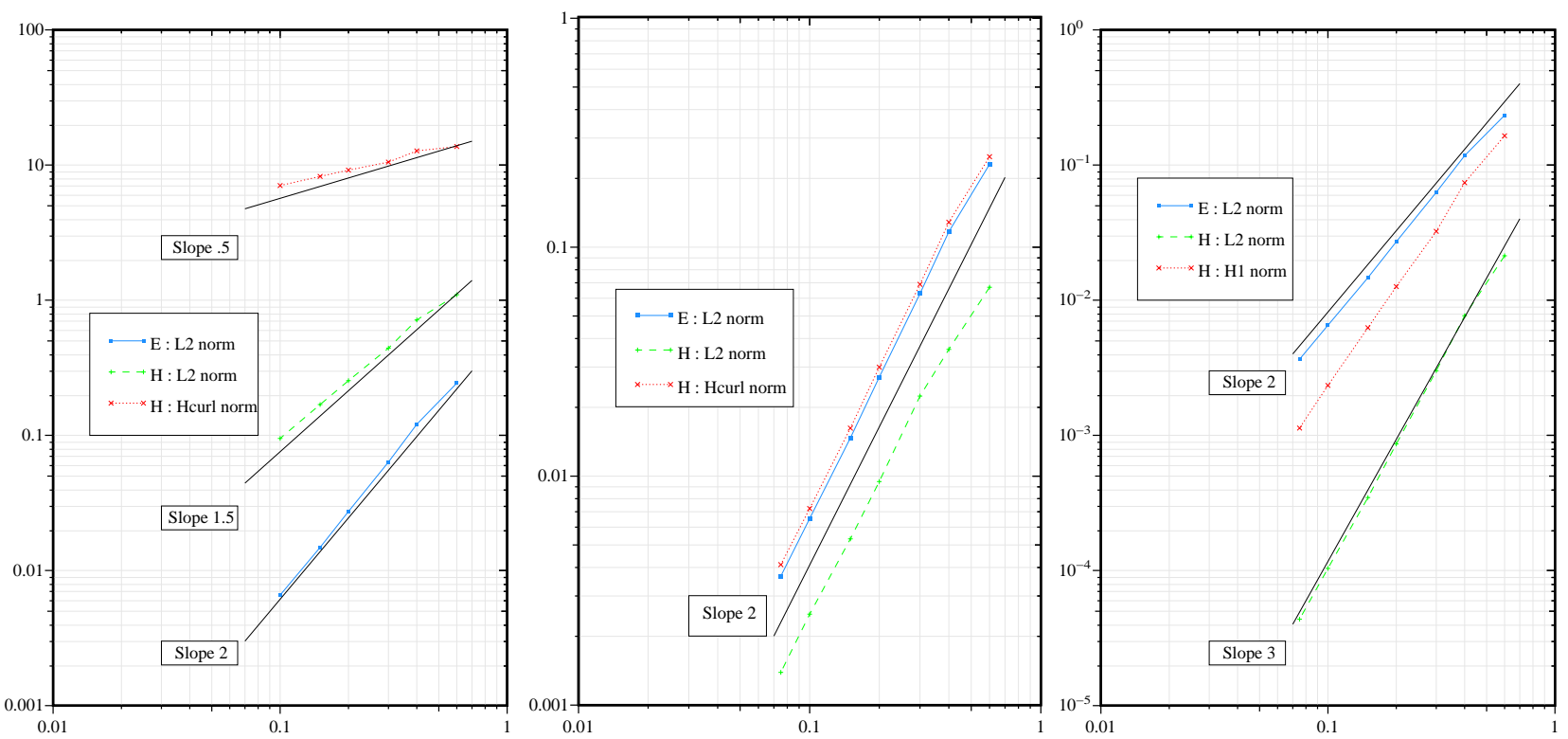

Figure 2. Convergence analysis. From left to right: bilinear form $a_{1}$; bilinear form $a_{1 s}$; bilinear form $a_{2}$.
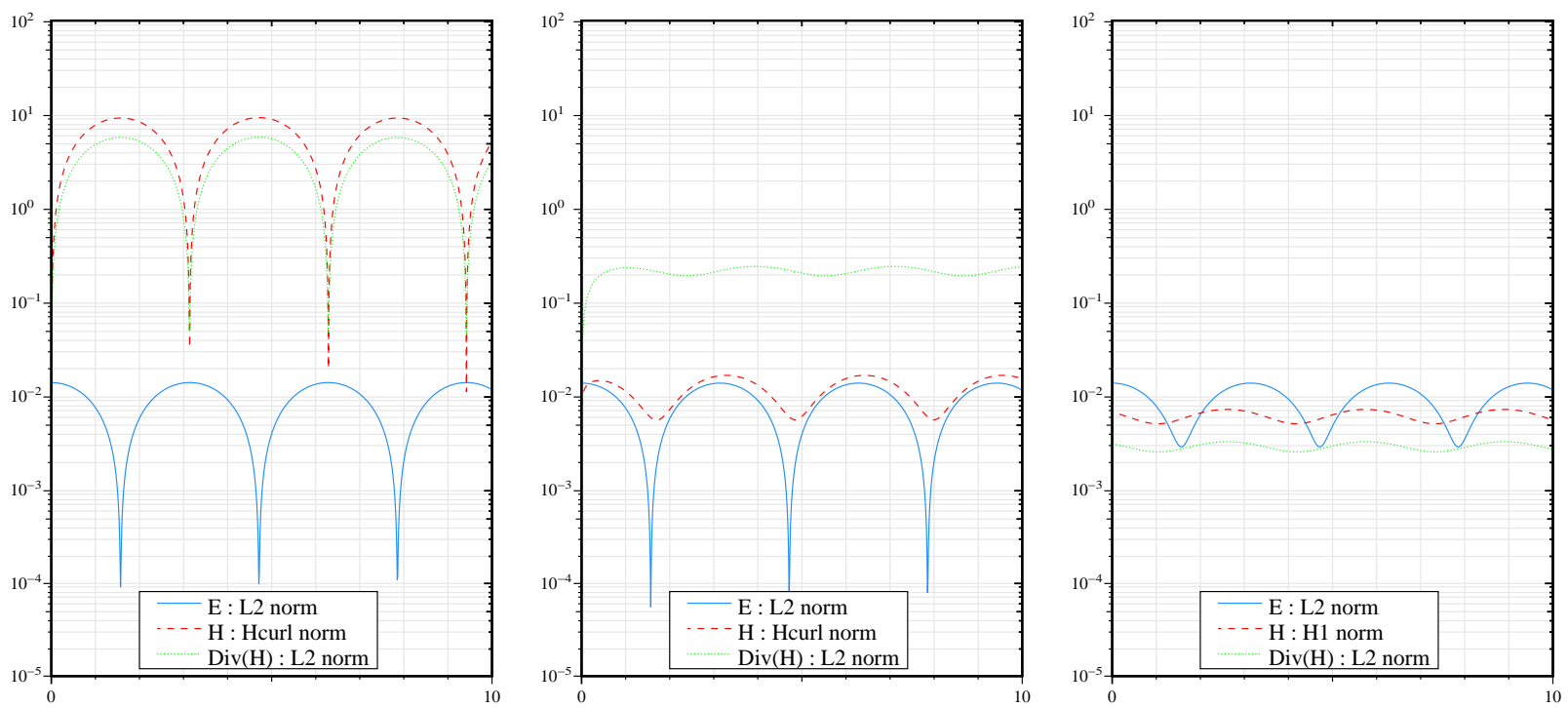

Figure 3. Time behavior of the error, $0 \leq t \leq 10$. From left to right: bilinear form $a_{1}$; bilinear form $a_{1 s}$; bilinear form $a_{2}$.

\subsection{A numerical illustration}

To illustrate the method we show some calculations for more realistic problems. We consider a conducting cylinder of radius $R=1$ embedded in a non-conducting domain so that

$$
\Omega=]-5 R,+5 R\left[, \quad \Omega_{c}=\left\{(x, y) \in \mathbb{R}^{2}, \sqrt{x^{2}+y^{2}}<R\right\} .\right.
$$



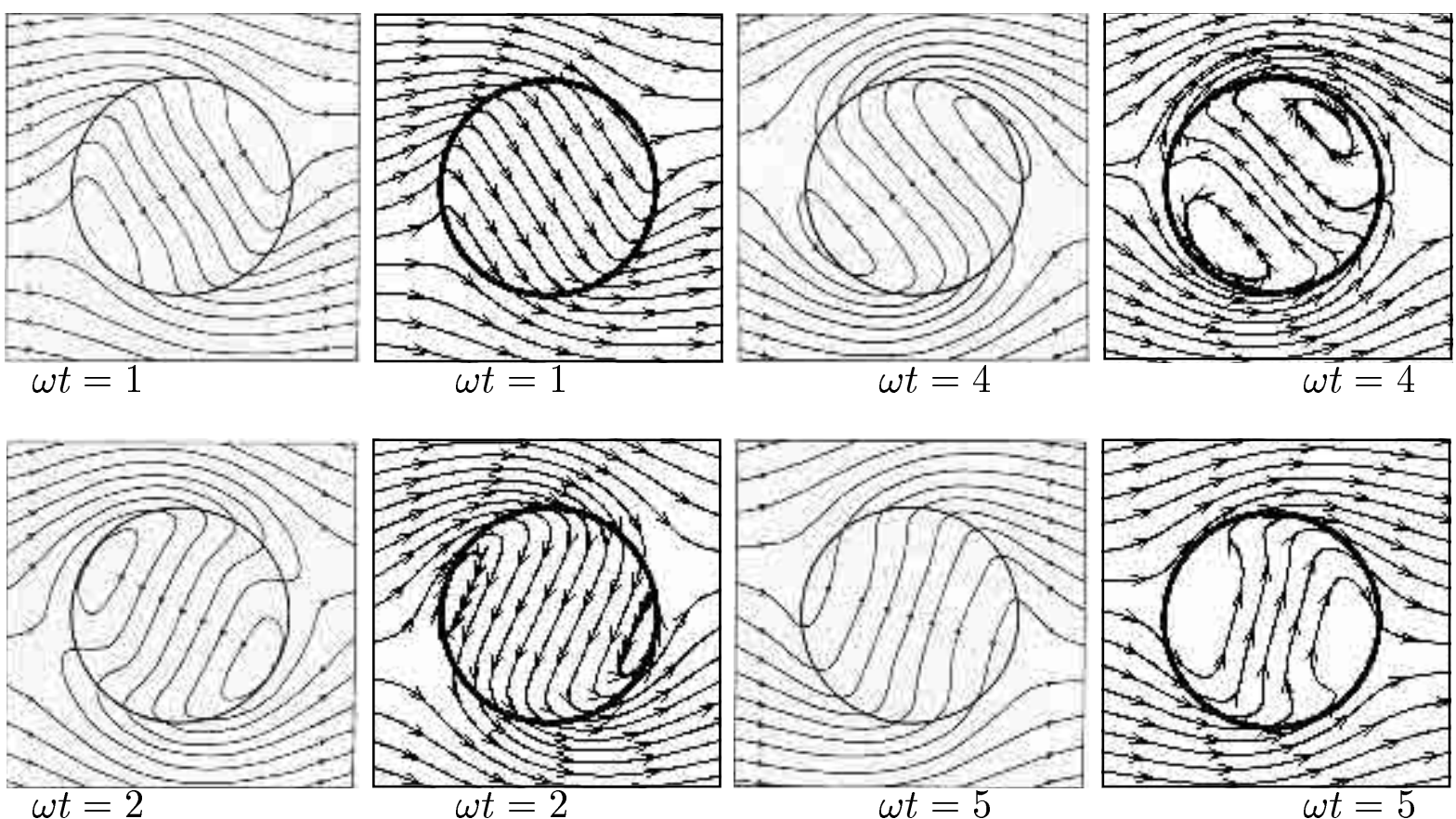

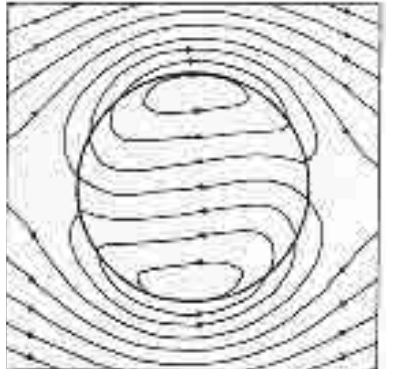

$\omega t=3$
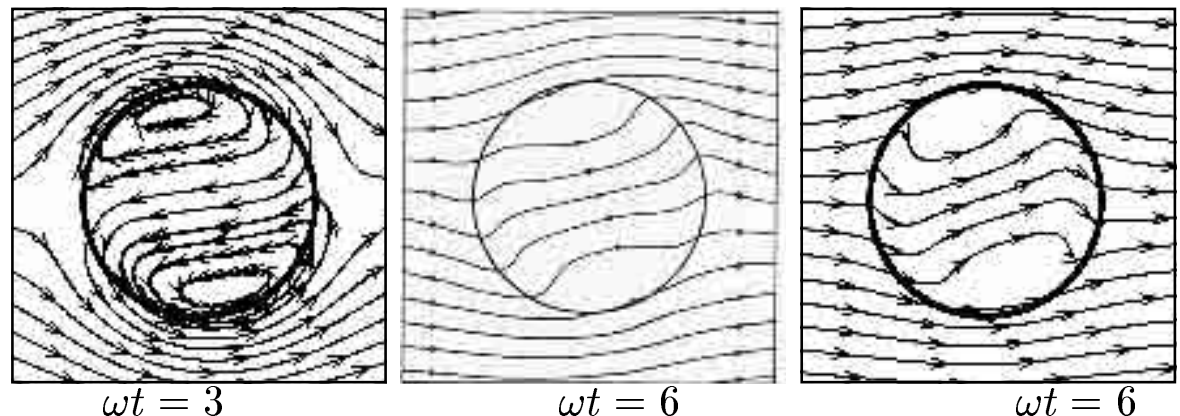

FiguRE 4. Streamlines of the magnetic field, $R_{m}=100$. Analytical solution from [20].

The magnetic permeability and the electric conductivity are constant. At $t \leq 0$ the cylinder is at rest; at latter times it rotates with the velocity $\mathbf{u}=\omega \mathbf{e}_{z} \times \mathbf{r}$, where $\mathbf{r}=(x, y, 0)$ and $\mathbf{e}_{z}=(0,0,1)$. This problem has been studied thoroughly in [20] and analytical solutions have been proposed.

Defining the magnetic Reynolds number $R_{m}=\mu \sigma \omega R^{2}$, we show in Figure 4 the streamlines of the magnetic field for $R_{m}=100$ at times $\omega t=1,2,3,4,5,6$. The analytical solution is plotted on the left of the figure and the $\mathbb{P}_{2} / \mathbb{P}_{1}$ solution is plotted on the right. Note the reconnection of the streamlines. A thorough qualitative analysis of this problem will be reported elsewhere [10].

To finish this paper we show in Figure 5 the steady state solutions for $R_{m}=10,20,40$, and 100 . Note the skin effect: as the magnetic Reynolds number increases the magnetic field is expelled from the conductor, and a boundary layer appears at the periphery of the cylinder. This effect is well captured by the numerical simulation. 

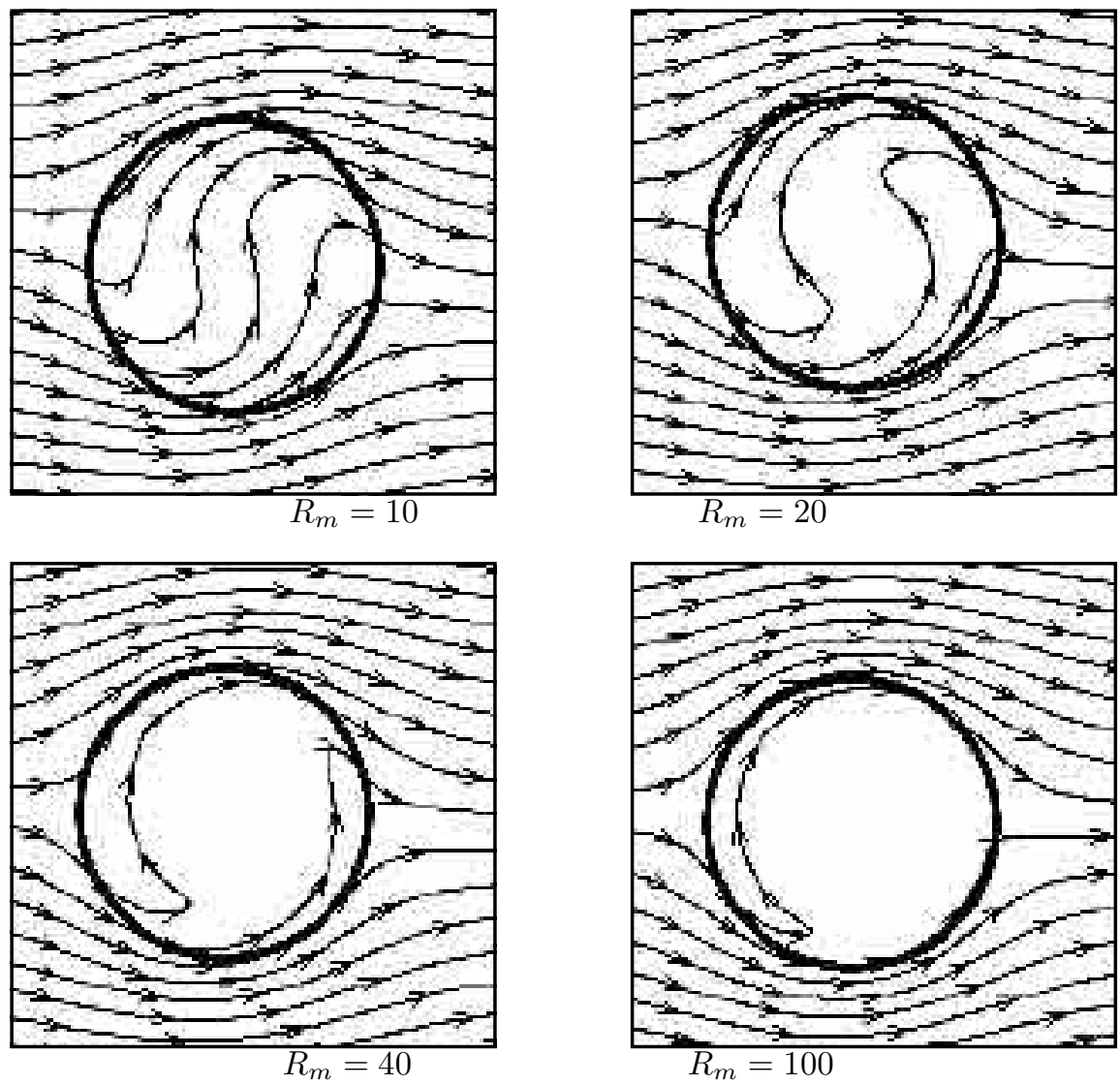

Figure 5. Streamlines of the magnetic field at steady state. Note the skin effect.

Acknowledgements. The calculations reported in Section 4.2 have been carried out by J. Messner and C. Nore at LIMSI, Orsay. The first author gratefully acknowledges the support of the Texas Institute for Computational and Applied Mathematics under a TICAM Visiting Faculty Fellowship. The second author would like to acknowledge the support via an NSERC research grant and a Visiting Fellowship from the University of Paris XI at Orsay. The authors wish to thank J. Léorat from the Observatoire de Paris-Meudon for bringing to their attention the problem considered in this paper and for his unflinching efforts to explain them MHD.

\section{REFERENCES}

[1] T. Amari, J.F. Luciani and P. Joly, A preconditioned semi-implicit method for magnetohydrodynamics equation. SIAM J. Sci. Comput. 21 (1999) 970-986.

[2] F. Brezzi and M. Fortin, Mixed and Hybrid Finite Element Methods. Springer Verlag, New York, Springer Ser. Comput. Math. 15 (1991).

[3] A. Bossavit, Electromagnétisme en vue de la modélisation. SMAI/Springer-Verlag, Paris, Math. Appl. 14 (1993). See also Computational Electromagnetism, Variational Formulations, Complementary, Edge Elements, Academic Press (1998).

[4] H. Brezis, Analyse fonctionnelle. Masson, Paris (1991).

[5] P. Clément, Approximation by finite element functions using local regularization. Anal. Numér. 9 (1975) 77-84.

[6] M. Costabel, A coercive bilinear form for Maxwell's equations. J. Math. Anal. Appl. 157 (1991) 527-541.

[7] M.L. Dudley and R.W. James, time-dependent kinematic dynamos with stationary flows. Proc. Roy. Soc. London A425 (1989) 407-429.

[8] L. Demkowicz and L. Vardapetyan, Modeling of electromagnetic absorption/scattering problems using $h p$-adaptive finite elements. Comput. Methods Appl. Mech. Engrg. 152 (1998) 103-124. Symposium on Advances in Computational Mechanics, Vol. 5 (Austin, TX, 1997). 
[9] J.-F. Gerbeau, A stabilized finite element method for the incompressible magnetohydrodynamic equations. Numer. Math. 87 (2000) 83-111.

[10] J.-L. Guermond, J. Léorat and C. Nore, Numerical simulations of 2D MHD problems using Lagrange finite elements (in preparation 2001).

[11] J.-L. Guermond and P.D. Minev, Mixed finite element approximation of an MHD problem involving conducting and insulating regions: the 3D case (submitted 2002).

[12] V. Girault and P.-A. Raviart, Finite Element Methods for Navier-Stokes Equations. Springer-Verlag, Berlin, Springer Ser. Comput. Math. 5 (1986).

[13] J. Léorat, Numerical simulations of cylindrical dynamos: scope and method. In 7th beer-Sheva Onternatal seminar, Vol. 162, pp. 282-292. AIAA Progress in Astronautics and aeronautic series, 1994.

[14] J. Léorat, Linear dynamo simulations with time dependent helical flows. Magnetohydrodynamics 31 (1995) 367-373.

[15] J.-L. Lions and E. Magenes, Problèmes aux limites non homogènes et applications, Vol. 1. Dunod, Paris (1968).

[16] H.K. Moffatt, Magnetic Field Generation in Electrically Conducting Fluids. Cambridge Monographs on Mechanics and Applied Mathematics. Cambridge University Press, Cambridge (1978).

[17] A.J. Meir and P.G. Schmidt, Analysis and numerical approximation of a stationary MHD flow problem with non-ideal boundary. SIAM J. Numer. Anal. 36 (1999) 1304-1332.

[18] J. Nečas, Les méthodes directes en théorie des équations elliptiques. Masson, Paris (1967).

[19] J.-C. Nédélec, A new family of mixed finite elements in $\mathbb{R}^{3}$. Numer. Math. 50 (1986) 57-81.

[20] R.L. Parker, Reconnexion of lines of force in rotating spheres and cylinders. Proc. Roy. Soc. 291 (1966) 60-72.

[21] N. Ben Salah, A. Soulaimani and W.G. Habashi, A finite element method for magnetohydrodynamics. Comput. Methods Appl. Mech. Engrg. 190 (2001) 5867-5892.

[22] N. Ben Salah, A. Soulaimani, W.G. Habashi and M. Fortin, A conservative stabilized finite element method for magnetohydrodynamics equations. Internat. J. Numer. Methods Fluids 29 (1999) 535-554.

[23] R. Verfürth, Error estimates for a mixed finite element approximation of the Stokes equation. RAIRO Anal. Numér. 18 (1984) $175-182$.

To access this journal online:

www.edpsciences.org 